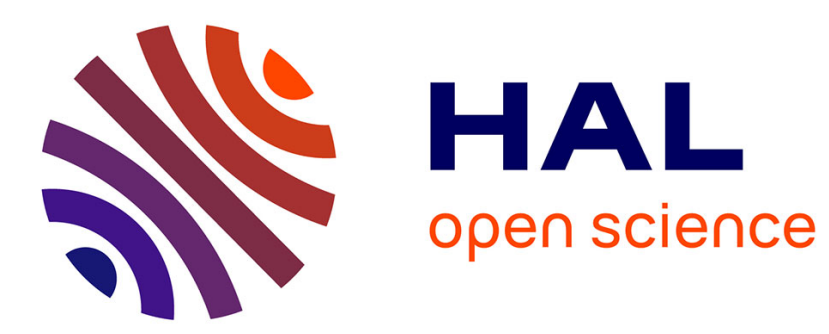

\title{
Modeling for non isothermal cavitation using 4-equation models
}

\author{
Eric Goncalvès da Silva
}

\section{To cite this version:}

Eric Goncalvès da Silva. Modeling for non isothermal cavitation using 4-equation models. International Journal of Heat and Mass Transfer, 2014, pp.1-17. 10.1016/j.ijheatmasstransfer.2014.04.065 . hal-00990670

\section{HAL Id: hal-00990670 https://hal.science/hal-00990670}

Submitted on 13 May 2014

HAL is a multi-disciplinary open access archive for the deposit and dissemination of scientific research documents, whether they are published or not. The documents may come from teaching and research institutions in France or abroad, or from public or private research centers.
L'archive ouverte pluridisciplinaire HAL, est destinée au dépôt et à la diffusion de documents scientifiques de niveau recherche, publiés ou non, émanant des établissements d'enseignement et de recherche français ou étrangers, des laboratoires publics ou privés. 


\title{
Modeling for non isothermal cavitation using four-equation models
}

\author{
Eric Goncalvès* \\ LEGI-University of Grenoble, 1025 rue de la Piscine, 38400 St Martin d'Heres, France
}

\begin{abstract}
A compressible, two-phase, one-fluid solver has been developed to investigate the behaviour of cavitation models including thermodynamic effects. The code is composed by three conservation laws for mixture variables (mass, momentum and total energy) and a supplementary transport equation for the void ratio. Two formulations for the mass transfer between phases are studied. Numerical simulations are firstly performed on rarefaction cavitating problems in which the working fluid is hot water and freon R-114. A realistic turbulent Venturi case with freon R-114 is performed and comparisons are done between 3- and 4-equation models. A warming effect is highlighted downstream the cavitation pocket in the region of pressure recuperation. Keywords: Cavitation, Homogeneous model, Mass transfer, Thermal effects
\end{abstract}

*Corresponding author.

Email address: Eric.Goncalves@legi.grenoble-inp.fr (Eric Goncalvès ) 


\section{Nomenclature}

$B \quad$ B-factor

c speed of sound

$C_{p}, C_{v} \quad$ thermal capacities

E total energy

e internal energy

$g \quad$ free Gibbs enthalpy

$h \quad$ enthalpy

$L_{v a p} \quad$ latent heat of vaporization

$\dot{m} \quad$ mass transfer between phases

$P \quad$ static pressure

$P_{\text {vap }} \quad$ vapour pressure

$P_{\infty} \quad$ reference pressure

$P_{r}, P_{r t} \quad$ molecular and turbulent Prandtl numbers

$Q \quad$ total heat flux

$q \quad$ energy of formation

$R e_{L} \quad$ Reynolds number based on the length $\mathrm{L}$

$T \quad$ temperature

$T_{\text {ref }} \quad$ reference temperature

$u, v \quad$ velocity components

$w \quad$ conservative variables

$Y \quad$ mass fraction of gas

$\alpha \quad$ volume fraction of gas

$\gamma \quad$ ratio of thermal capacities

$\lambda, \lambda_{t} \quad$ molecular and turbulent thermal conductivity 


$\begin{array}{ll}\mu, \mu_{t} & \text { molecular and eddy viscosity } \\ \rho & \text { density } \\ \sigma & \text { cavitation number } \\ \tau & \text { total stress tensor } \\ ()_{l} & \text { liquid value } \\ ()_{v} & \text { vapour value } \\ ()^{s a t} & \text { saturation value } \\ ()^{v} & \text { viscous } \\ ()^{t} & \text { turbulent }\end{array}$

\section{Introduction}

Cavitation is a significant engineering phenomenon that occurs in fluid machinery, fuel injectors, marine propellers, nozzles, underwater bodies, etc. In most cases, cavitation is an undesirable phenomenon, significantly degrading performance, resulting in reduced flow rates, lower pressure increases in pumps, load asymmetry, vibrations, noise and erosion. Such flows are characterized by important variations of the local Mach number (due to the drastic diminution of the speed of sound in the mixture), large density ratio between the liquid and the vapor phases, compressibility effects and non equilibrium thermodynamic states.

Cavitation can be manifested at a constant temperature, and thus, it is usually assumed to be an isothermal phenomenon. However, the constant temperature hypothesis is no longer valid when cryogenic fluids (also known as thermosensitive fluids) are considered. For such fluids, the liquid-vapour density ratio is lower than 
that of typical fluids (cold water) and consequently more liquid mass has to vaporize to sustain a cavity. Therefore evaporative cooling effects are more pronounced and the temperature of the liquid in the immediate vicinity of the liquid-vapour interface is depressed below the free-stream temperature. Because of the strong variation of thermodynamic properties (vapour pressure, density), the temperature depression, negligible in water, is quite substantial. The local cooling effect delays the cavitation phenomenon and reduces the local vapour pressure of the fluid, which leads to a lower observed cavity pressure.

Several physical and numerical models have been developed to investigate cavitating flows within the framework of averaged two-phase model or bubbly models based on the Rayleigh-Plesset equation. For the averaged model, there are different approaches according to the assumptions made on the local thermodynamic equilibrium and the slip condition between phases. A hierarchy of models exists, with the numbers of equations ranging from seven to three only. The full non-equilibrium seven-equation models are the most complete. For both fluids, it contains equations for the mass, momentum and energy, and the seventh equation describes the topology of the flow. These models can take into account the physical details occurring in the cavitation phenomenon such as mass exchange, thermal transfer and surface tension. However, the transfer terms have to be known; such quantities are usually very difficult to obtain. Various formulations have been investigated to deal with metastable states and evaporation front dynamics $[1,2,3,4,5]$. Temperature and free Gibbs enthalpy exchange terms are included in the equations as relaxation terms to model heat and mass transfer. For thermal-hydraulics applications with cavitation, nucleation and boiling flows, a six-equation model has been developed [6, 7]. The interfacial mass transfer is modeled as a function of the interfacial heat transfer terms and the inter- 
facial phase-averaged enthalpies.

A reduced five-equation model can be derived with the assumptions of velocity equilibrium and pressure equilibrium. The archetype five-equation model is that of Kapila [8]. It is composed of four conservation laws: two for masses, one for the mixture momentum and one for the mixture energy. It is completed by an equation for a non-conservative quantity describing the flow topology, usually the void ratio. Such a model has been used for inviscid high speed cavitating applications and cavitation pocket in fuel injector nozzles $[9,10]$. Heat and mass transfer processes are modelled through thermal and chemical relaxation procedures.

By assuming the thermal equilibrium between phases, a four-equation model can be expressed. A very popular formulation, originally developed to simulate turbulent cavitating flows in cold water, has been adapted to cryogenic applications $[11,12,13,14]$. It is composed by three conservation laws for mixture quantities (mass, momentum, energy) plus a mass equation for the vapour or liquid density including a cavitation source term. The main difficulty is related to the formulation of the source term and the tunable parameters involved for the vaporization and condensation processes (different sets of parameters are presented in [12]). Moreover, this family of models are not thermodynamically well-posed and does not respect thermodynamic constraints [15]. Another popular model devoted to ebullition problems uses a mass fraction equation with a relaxation term (Homogeneous Relaxation Model). The source term involves a relaxation time that is the time for the system to regain its thermodynamic equilibrium state. This time is difficult to determine and is estimated from experimental data $[16,17,18,19]$.

With the assumption of complete thermodynamic equilibrium between phases (local temperature, pressure and free Gibbs enthalpy equality between phases), we obtain the 3-equation models or Homogeneous Equilibrium Models (HEM). Vaporization 
or condensation processes are assumed to be instantaneous. An equation of state (EOS) is necessary to close the system. Different closure relations (tabulated EOS or combination of pure phase EOSs) that link the pressure to the thermodynamic variables have been proposed [20, 21, 22, 23, 24].

The bubbly flow models are composed by three balance equations for the mixture quantities coupled with a macroscopic model for the bubble dynamics based on the Rayleigh-Plesset equation. This model is capable of handling either single bubbles or clouds of bubbles that grow and decrease through a pressure field $[25,26,27]$. In the case where heat transfer is negligible, the phase change is driven by inertia effects. Yet, when thermal effects are involved, the liquid inertia become rapidly negligible and the evolution is controlled by the heat flux provided by the liquid at the bubble surface. By comparing characteristic times of thermal and inertial phenomena, it can be shown that this thermal regime is an accurate representation of reality for moderate levels of superheating or subcooling [28, 29, 30].

In a recent study, we proposed a new mass transfer formulation associated to a fourequation model for isothermal cavitation [31, 32]. The generic formulation involves the ratio $c^{2} / c_{\text {wallis }}^{2}$ between the mixture speed of sound and the Wallis velocity, which is the speed of sound without heat and mass transfer. First, we extend the isothermal formulation with a non isothermal thermodynamic path using a linear approximation of the vapour pressure evolution. This model is built using the mixture speed of sound evaluated with a modified barotropic equation of state [24]. A second closure is investigated using a mixture of stiffened gas EOS and its associated mixture speed of sound. The validation is done through one-dimensional inviscid double rarefaction test cases in which reference solutions have been computed [5]. A new test case is 
proposed with the thermosensitive freon R-114 $\left(C_{2} C l_{2} F_{4}\right)$ as working fluid. Secondly, models are compared with experimental data on a turbulent Venturi case in which the running fluid is freon R-114. Local analyses with void ratio profiles and wall temperature depression are proposed. A warming effect downstream the cavitation pocket is exhibited.

This paper is organized as follows. We give a brief description of models. The averaged Navier-Stokes equations are presented and the numerical methods are described. Numerical results are presented with comparisons between models and validations against two-fluid solutions. The study of the turbulent venturi case is described. Finally, conclusions and future investigations are discussed.

\section{Mixture models and mass transfer}

The numerical simulations are carried out using an in-house CFD code solving the one-fluid compressible Euler and Navier-Stokes systems.

The homogeneous mixture approach is used to model two-phase flows. The phases are assumed to be sufficiently well mixed and the disperse particle size are sufficiently small thereby eliminating any significant relative motion. The phases are strongly coupled and moving at the same velocity. In addition, the phases are assumed to be in thermal and mechanical equilibrium: they share the same temperature $T$ and the same pressure $P$. The evolution of the two-phase flow can be described by the conservation laws that employ the representative flow properties as unknowns just as in a single-phase problem.

We introduce $\alpha$ the void fraction or the averaged fraction of presence of the vapour. The density $\rho$, the center of mass velocity $u$ and the internal energy $e$ for the mixture 
are defined by [33]:

$$
\begin{aligned}
\rho & =\alpha \rho_{v}+(1-\alpha) \rho_{l} \\
\rho u & =\alpha \rho_{v} u+(1-\alpha) \rho_{l} u \\
\rho e & =\alpha \rho_{v} e_{v}+(1-\alpha) \rho_{l} e_{l}
\end{aligned}
$$

To close the system, an equation of state (EOS) is necessary to link the pressure and the temperature to the internal energy and the density. For the pure phases, we used the convex stiffened gas EOS (see [34]):

$$
\begin{aligned}
P(\rho, e) & =(\gamma-1) \rho(e-q)-\gamma P_{\infty} \\
P(\rho, T) & =\rho(\gamma-1) C_{v} T-P_{\infty} \\
T(\rho, h) & =\frac{h-q}{C_{p}}
\end{aligned}
$$

where $\gamma=C_{p} / C_{v}$ is the heat capacity ratio, $C_{p}$ and $C_{v}$ are thermal capacities, $q$ the energy of the fluid at a given reference state and $P_{\infty}$ is a constant reference pressure. The speed of sound $c$ is given by:

$$
c^{2}=\gamma \frac{P+P_{\infty}}{\rho}=(\gamma-1) C_{p} T
$$

For the mixture, two EOS are tested: a mixture of stiffened gas (SG) and a modified barotropic EOS. 


\subsection{A mixture of stiffened gas EOS}

By assuming the pressure equilibrium between phases, an expression for the pressure can be deduced, function of the void ratio $\alpha$ and the vapour mass fraction $Y=\alpha \rho_{v} / \rho$ :

$$
\begin{aligned}
P(\rho, e, \alpha, Y) & =(\gamma(\alpha)-1) \rho(e-q(Y))-\gamma(\alpha) P_{\infty}(\alpha) \\
\frac{1}{\gamma(\alpha)-1} & =\frac{\alpha}{\gamma_{v}-1}+\frac{1-\alpha}{\gamma_{l}-1} \\
q(Y) & =Y q_{v}+(1-Y) q_{l} \\
P_{\infty}(\alpha) & =\frac{\gamma(\alpha)-1}{\gamma(\alpha)}\left[\alpha \frac{\gamma_{v}}{\gamma_{v}-1} P_{\infty}^{v}+(1-\alpha) \frac{\gamma_{l}}{\gamma_{l}-1} P_{\infty}^{l}\right]
\end{aligned}
$$

By assuming the thermal equilibrium between phases, the mixture temperature is expressed as:

$$
\begin{aligned}
T(\rho, h, Y) & =\frac{h_{l}-q_{l}}{C_{p_{l}}}=\frac{h_{v}-q_{v}}{C_{p_{v}}}=\frac{h-q(Y)}{C_{p}(Y)} \\
C_{p}(Y) & =Y C_{p_{v}}+(1-Y) C_{p_{l}}
\end{aligned}
$$

We assume that the vaporization pressure varies linearly with the temperature:

$$
P_{v a p}(T)=P_{v a p}\left(T_{r e f}\right)+\frac{d P}{d T}\left(T-T_{r e f}\right)
$$

The constant quantity $d P / d T$ is evaluated with a thermodynamic table.

The speed of sound in the mixture can be expressed as a function of the enthalpy of each phase (see Appendix A):

$$
\begin{aligned}
C_{1} & =\frac{1}{\gamma-1}+\frac{\rho_{v} h_{v}-\rho_{l} h_{l}}{\left(\rho_{l}-\rho_{v}\right) \frac{d P}{d T}}\left(\alpha \frac{d \rho_{v}}{d T}+(1-\alpha) \frac{d \rho_{l}}{d T}\right) \\
\rho c^{2} & =\frac{1}{C_{1}}\left[\frac{\rho_{v} \rho_{l}}{\left(\rho_{l}-\rho_{v}\right)}\left(h_{v}-h_{l}\right)\right]
\end{aligned}
$$

Enthalpies of pure phase $h_{l}$ and $h_{v}$ are computed with the mixture temperature $T$. Due to numerical problems, the derivatives of densities with the temperature are not taken into account in this study. 


\subsection{A modified barotropic EOS}

A modified barotropic relation [24] can be considered for the mixture. When the pressure is between $P_{\text {vap }}(T)+\Delta P$ and $P_{\text {vap }}(T)-\Delta P$, the following relationship applies:

$$
P(\alpha, T)=P_{\text {vap }}(T)+\left(\frac{\rho_{l}^{s a t}-\rho_{v}^{s a t}}{2}\right) c_{\text {baro }}^{2} \operatorname{Arcsin}(1-2 \alpha)
$$

where $\Delta P$ represents the pressure range of the law and, for a void ratio value of 0.5 , the pressure is equal to the saturation pressure $P_{\text {vap }}(T)$ at the local temperature $T$. This temperature is evaluated using the relation (13). $\rho_{l}^{\text {sat }}$ and $\rho_{v}^{\text {sat }}$ are pure phases densities at saturation evaluated at the reference temperature $T_{r e f}$. The quantity $c_{\text {baro }}$, which has the dimension of a velocity, is a parameter of the model.

As previously we assume that the vaporization pressure varies linearly with the temperature and follows the relation (14).

The speed of sound in the mixture can be written as [24]:

$$
\begin{aligned}
c^{2} & =\frac{\frac{\rho_{\rho_{V} \rho_{L}}}{\rho\left(\rho_{L}-\rho_{V}\right)}\left(h_{V}-h_{L}\right) \frac{d P}{d T}+\rho C_{p} c_{T}^{2}}{\rho C_{p}-\frac{d P}{d T}} \\
C_{p} & =Y C_{p_{v}}+(1-Y) C_{p_{l}} \\
c_{T}^{2} & =\left(\frac{\partial P}{\partial \rho}\right)_{s}=\left(\frac{\partial P}{\partial \rho}\right)_{T}=\frac{c_{\text {baro }}^{2}}{2 \sqrt{\alpha(1-\alpha)}}
\end{aligned}
$$

Where $c_{T}$ is the isothermal speed of sound (i.e. when $d P / d T=0$ ).

\subsection{A void ratio transport-equation with mass transfer}

The model consists in three conservation laws for mixture quantities and an additional equation for the void ratio. It is obtained from a reduction of the Kapila 
five-equation model assuming the liquid is at its saturation state [31]. We present below only the void ratio equation:

$$
\frac{\partial \alpha}{\partial t}+V \frac{\partial \alpha}{\partial x}=\underbrace{\left(\frac{\rho_{l} c_{l}^{2}-\rho_{v} c_{v}^{2}}{\frac{\rho_{l} c_{l}^{2}}{1-\alpha}+\frac{\rho_{v} c_{v}^{2}}{\alpha}}\right)}_{=K} \operatorname{div} V+\underbrace{\left(\frac{\frac{c_{v}^{2}}{\alpha}+\frac{c_{l}^{2}}{1-\alpha}}{\frac{\rho_{l} c_{l}^{2}}{1-\alpha}+\frac{\rho_{v} c_{v}^{2}}{\alpha}}\right)}_{=1 / \rho_{I} \text { the interfacial density }} \dot{m}
$$

where $\dot{m}$ denotes the mass transfer between phases, $V$ the homogeneous velocity vector, $c_{k}$ the speed of sound of the phase $k$.

By assuming that the mass transfer is proportional to the velocity divergence, it is possible to build a family of models in which the mass transfer $\dot{m}$ is expressed as

$$
\dot{m}=\frac{\rho_{l} \rho_{v}}{\rho_{l}-\rho_{v}}\left(1-\frac{c^{2}}{c_{\text {wallis }}^{2}}\right) \operatorname{div} V
$$

where $c_{\text {wallis }}$ is the propagation velocity of acoustic waves without mass transfer [35]. This speed of sound is expressed as a weighted harmonic mean of speeds of sound of each phase:

$$
\frac{1}{\rho c_{\text {wallis }}^{2}}=\frac{\alpha}{\rho_{v} c_{v}^{2}}+\frac{1-\alpha}{\rho_{l} c_{l}^{2}}
$$

The liquid density $\rho_{l}$ is assumed to be in its equilibrium state at the reference temperature: $\rho_{l}=\rho_{l}^{s a t}\left(T_{r e f}\right)$. We did not test to introduce a thermal variation for the liquid density. The vapour density $\rho_{v}$ follows the pure phase EOS (i.e. the stiffened gas EOS) and varies with the temperature.

With this generic form for the mass transfer, we remark that all models in which the mixture speed of sound is the Wallis one can not produce or destroy the void ratio during the phase transition (it is the case of most of void ratio transport equation models). The void ratio is only modified through the term $K$ involving the compressibility of pure phases. 


\subsection{Model with a barotropic EOS}

A first model is built using the speed of sound of the modified barotropic model presented previously. In the following, this model will be named 4-equation barotropic model. It involves two parameters: $c_{b a r o}$ and $d P / d T$.

As the temperature and pressure relations are coupled (relations 14 and 17), an iterative procedure on the temperature is introduced. The temperature is initialized by the liquid temperature computed with the pure phase EOS. Five iterations are done and it has been checked that the numerical solution did not change with a higher number of iteration.

Without thermal variation, this model has been successfully tested on rarefaction tube problems [31] and cavitating venturi flows [32].

\subsection{Model with a mixture of stiffened gas EOS}

A second model is built using the mixture of stiffened gas EOS. The mass transfer term is activated when the local pressure $P$ is smaller than the vapour pressure $P_{v a p}(T)$ evaluated using the relation (14). This model will be named 4-equation SG model. It involves only the parameter $d P / d T$.

As previously, the temperature and pressure relations are coupled and an iterative procedure on the temperature is done with five iterations. It has been checked that the numerical solution did not change with a higher number of iteration.

Characteristics of both four-equation models and the three-equation barotropic model [24] are summarized in Table 8. In addition, characteristics of the two-fluid model 
of Zein et al. [5] based on relaxation procedures on the pressure, temperature and Gibbs enthalpy are presented.

\subsection{Speed of sound in the mixture}

The mass transfer formulation involves explicitly the difference between the speed of sound in the mixture and the Wallis one. Fig. 1 compares the evolution of this speed of sound (with a logarithmic scale) as a function of the void ratio for the EOS presented, the Wallis formulation and the equilibrium speed of sound in a mixture of freon R-114. Without heat and mass transfer effects, the propagation of acoustic waves follows the Wallis speed of sound. When exchanges of mass and heat between phases are involved, the sound speed decreases to the thermodynamic equilibrium one [36]. This limit speed is evaluated with the assumption of local thermodynamic equilibrium between phases: equalities of pressure, temperature and free Gibbs enthalpy between phases. The expression of this velocity is given in [15]. To compare the different speeds, the temperature is assumed to be constant equal to $293 \mathrm{~K}$ and saturation values are used. The parameters of the stiffened gas EOS are given in table 2. For the barotropic EOS, the parameter $c_{\text {baro }}$ is set at $1.5 \mathrm{~m} / \mathrm{s}$. We can observe that for both EOS the mixture speed of sound is well limited by the Wallis and the equilibrium ones. Both models are able to create vapour as soon as the divergence of velocity is strictly positive. Moreover, we remark that the barotropic speed of sound is very close to the equilibrium one while the SG speed is relatively close to the Wallis one.

\section{Reynolds averaged Navier-Stokes equations}

For turbulent computations, the compressible one-fluid RANS equations are used, coupled with the one-equation turbulence model of Spalart-Allmaras (SA) [37]. These 
equations can be expressed as:

$$
\begin{gathered}
\frac{\partial w}{\partial t}+\operatorname{div}\left(F_{c}-F_{v}\right)=S \\
w=\left(\begin{array}{c}
\rho \\
\rho V \\
\rho E \\
\alpha \\
\rho \tilde{\nu}
\end{array}\right) \quad ; \quad F_{c}=\left(\begin{array}{c}
\rho V \\
\rho V \otimes V+P \overline{\bar{I}} \\
(\rho E+P) V \\
\alpha V \\
\rho \tilde{\nu} V
\end{array}\right) \quad ; F_{v}=\left(\begin{array}{c}
0 \\
\overline{\overline{\tau^{v}}}+\overline{\overline{\tau^{t}}} \\
\left(\overline{\overline{\tau^{v}}}+\overline{\overline{\tau^{t}}}\right) \cdot V-Q^{v}-Q^{t} \\
0 \\
\left(\mu+\rho \tilde{\nu} / \sigma_{\nu}\right) \operatorname{grad} \tilde{\nu}
\end{array}\right)
\end{gathered}
$$

where $w$ denotes the conservative variables and the void ratio, $F_{c}$ and $F_{v}$ the convective and viscous flux densities and $S$ the source terms, which concern the void ratio equation and the turbulent transport equation. $E=e+u^{2} / 2$ is the mixture total energy. $\tilde{\nu}$ is the transported turbulent variable.

The total stress tensor $\overline{\bar{\tau}}$ is evaluated using the Stokes hypothesis, Newton's law and the Boussinesq assumption. The total heat flux vector $Q$ is obtained from the Fourier law involving a turbulent thermal conductivity $\lambda_{t}$ with the constant Prandtl number hypothesis.

$$
\begin{aligned}
& \overline{\bar{\tau}}=\overline{\overline{\tau^{v}}}+\overline{\overline{\tau^{t}}}=\left(\mu+\mu_{t}\right)\left[\left(\operatorname{grad} V+(\operatorname{grad} V)^{t}\right)-\frac{2}{3}(\operatorname{div} V) \overline{\bar{I}}\right]+\frac{2}{3} \rho k \overline{\bar{I}} \\
& Q=Q^{v}+Q^{t}=-\left(\lambda+\lambda_{t}\right) \operatorname{grad} T \quad \text { with } \quad \lambda_{t}=\frac{\mu_{t} C_{p}}{P_{r t}}
\end{aligned}
$$

In the pure liquid, the viscosity is determined by an exponential law and, in pure vapour, the viscosity follows the Sutherland law. The mixture viscosity is defined as the arithmetic mean of the liquid and vapour viscosities (fluctuations of viscosity 
are neglected) [33]:

$$
\begin{aligned}
\mu_{L}(T) & =\mu_{0_{L}} \exp (B / T) \\
\mu_{V}(T) & =\mu_{0_{V}} \sqrt{\frac{T}{293}} \frac{1+T_{S} / 293}{1+T_{S} / T} \\
\mu(T, \alpha) & =\alpha \mu_{V}(T)+(1-\alpha) \mu_{L}(T)
\end{aligned}
$$

where $\mu_{0_{L}}, \mu_{0_{V}}, B$ and $T_{S}$ are constant parameters.

The mixture thermal conductivity $\lambda$ is also defined as the arithmetic mean of the liquid and vapour values:

$$
\lambda(\alpha, T)=\alpha \frac{\mu_{V}(T) C_{p_{V}}}{P_{r_{V}}}+(1-\alpha) \frac{\mu_{L}(T) C_{p_{L}}}{P_{r_{L}}}
$$

The turbulent Prandtl number $P_{r t}$ is set to 1 .

For the modelling of flow close to the wall, a two-layer wall law approach is used [38].

\section{Numerical methods}

The numerical simulations are carried out using an explicit CFD code based on a finite-volume discretization. For the mean flow, the convective flux density vector on a cell face is computed with the Jameson-Schmidt-Turkel scheme [39]. The artificial viscosity includes a second-order dissipation term $D_{2}$ and a fourth-order dissipation term $D_{4}$, which involve two tunable parameters $k^{(2)}$ and $k^{(4)}$.

The viscous terms are discretized by a second-order space-centered scheme. For the turbulence transport equations, the upwind Roe scheme [40] is used to obtain a more robust method. The second-order accuracy is obtained by introducing a flux-limited dissipation [41]. 
The numerical treatment of boundary conditions is based on the use of the characteristic relationships. More details are given in [31].

\section{1D inviscid rarefaction test case with water}

A double rarefaction tube problem is considered with an initial velocity discontinuity located at the middle of the tube. This test consists in a one meter long tube filled with liquid water at atmospheric pressure and with density $\rho_{l}=1150 \mathrm{~kg} / \mathrm{m}^{3}$. The temperature of water is $T_{r e f}=355 \mathrm{~K}$. A weak volume fraction of vapor $\alpha=0.01$ is initially added to the liquid. The initial discontinuity is set at $0.5 \mathrm{~m}$, the left velocity and the right velocity have the same value but an opposite sign. Two velocities are tested: $u= \pm 2 \mathrm{~m} / \mathrm{s}$ and $u= \pm 100 \mathrm{~m} / \mathrm{s}$. The vapour pressure $P_{\text {vap }}\left(T_{r e f}\right)$ is set to $51000 \mathrm{~Pa}$ at the initial temperature.

The solution involves two expansion waves. As gas is present, the pressure cannot become negative. To maintain positive pressure, the gas volume fraction increases due to the gas mechanical expansion and creates a pocket. Liquid water is expanded until the saturation pressure is reached then evaporation appears and quite small amount of vapor is created. The solution with phase transition is composed of four expansion waves. The extra two expansion waves correspond to the evaporation fronts.

These cases were computed in [5] using a two-fluid model in which instantaneous relaxation processes toward equilibrium are included for the temperature and the Gibbs free energy. The mesh contains 5000 cells. The time step is set to $10^{-7} \mathrm{~s}$. The value of $c_{\text {baro }}$ is set to $1.31 \mathrm{~m} / \mathrm{s}$ for the 4-equation barotropic model as presented in [31].

The parameters of the stiffened gas EOS and saturation values for densities are given in Table 3. The quantities have been evaluated with a saturation table at the 
reference temperature.

\subsection{Velocity $|u|=2 \mathrm{~m} / \mathrm{s}$}

The value of $d P / d T$, evaluated with a thermodynamic table on the temperature interval $[350 K, 355 K]$, is set to $2000 \mathrm{~Pa} / \mathrm{K}$. Void ratio, velocity, temperature, pressure, speed of sound and mass fraction of gas evolutions are shown in Fig. 2 at time $t=3.2$ ms. Results are compared with the two-fluid solution computed by Zein et al. [5]. Both models provide a similar evolution for the pressure, velocity, volume and mass fraction of gas, in good agreement with the two-fluid solution. The mixture speeds of sound for both models are compared with the Wallis formulation (with a logarithmic scale). A large discrepancy is noticeable between models. Using the SG model, the speed of sound is equal to the Wallis one excepted in the cavitation area where it is a little smaller. This small gap is enough to create the cavitation pocket. On the contrary, the speed of sound given by the barotropic model is clearly lower than the Wallis one (by a factor 5), as observed in Figure 1.

The temperature evolution puts in evidence the small cooling effect during the phase transition. For this test case, the thermal effect is weak. The temperature drop reaches $0.25 \mathrm{~K}$ with the 4-equation barotropic model and $0.5 \mathrm{~K}$ with the 4-equation SG model. Unfortunately the temperature evolution was not plotted in [5].

A simple heat balance between the two phases can estimate the scale of temperature difference $\Delta T^{*}$ caused by thermal effects. The $B$-factor is estimated as the ratio between the actual temperature drop and $\Delta T^{*}$ :

$$
\Delta T^{*}=\frac{\rho_{v} L_{v a p}}{\rho_{l} C_{p_{l}}} \quad \text { and } \quad B=\frac{\Delta T}{\Delta T^{*}}
$$

where $L_{v a p}$ is the latent heat and $C_{p_{L}}$ represents the specific heat. 
The B-factor of ideal gas-liquid mixture is expressed by the following equation [42]:

$$
B=\frac{\alpha}{1-\alpha}
$$

With the maximum value reached during the computation, that is $\alpha=0.7$, the ideal temperature depression is around $0.33 \mathrm{~K}$. Both models provide a cooling effect close to this value.

\subsection{Velocity $|u|=100 \mathrm{~m} / \mathrm{s}$}

The same conditions are used except regarding velocities which are set to $|u|=100$ $\mathrm{m} / \mathrm{s}$. In this case, evaporation is much more intense resulting in a large cavitation pocket where the gas volume fraction is close to 1 . However, this pocket does not contain pure gas but a mixture at thermodynamic equilibrium. For this test case, thermal effects are strong.

The value of $d P / d T$, evaluated with a thermodynamic table on the temperature interval $[250 K, 355 K]$, is set to $300 \mathrm{~Pa} / \mathrm{K}$. The volume and mass fractions of gas, pressure, temperature, velocity and speed of sound evolutions are plotted in Fig. 3, at time $t=1.5 \mathrm{~ms}$. No differences appear on the void ratio between models whereas the mass fraction simulated by our models is twice higher in comparison with the two-fluid solution. It is due to differences in the evaluation of the gas density. In our models, $\rho_{v}$ is function of the mixture temperature through the stiffened gas EOS while the gas density is function of the gas temperature for the two-fluid model. For the pressure profiles, the pressure drop under $P_{\text {vap }}\left(T_{r e f}\right)$ is around 0.3 bar with both models, in close agreement with the two-fluid solution. Discrepancies appear for the velocity profile in comparison with the two-fluid solution: variations across the evaporation front are stiffer with our models. As previously observed, the SG 
speed of sound is slightly smaller than the Wallis one near the evaporation fronts. Inside the cavitation pocket, the barotropic speed of sound is close to the Wallis one whereas the SG velocity is twice lower.

The temperature drop inside the cavitation pocket due to the phase transition is very large. It reaches more than $70 \mathrm{~K}$ with the 4-equation SG model and $90 \mathrm{~K}$ with the 4-equation barotropic model.

For this case, the difference with the isothermal solution presented in [31] is very strong. Solutions between isothermal and non isothermal 4-equation barotropic models are illustrated in Fig. 4. For the pressure profiles, the pressure drop under $P_{\text {vap }}\left(T_{\text {ref }}\right)$ is around 0.05 bar with the isothermal model. Moreover the temperature drop is very intense and lead to negative values.

\subsection{Shock-cavitation interaction, $|u|=100 \mathrm{~m} / \mathrm{s}$}

This case is similar to the previous one, except that the two ends of the tube are simultaneously closed once the flow starts. Therefore, a shock created at each end moves towards the center, resulting in shock-cavitation interaction and cavitation collapse. Both evaporation and condensation processes can be investigated.

The flow is initially quasi pure water (the initial fraction of gas is $10^{-10}$ ) and soon changes phase into a vapour-liquid mixture at the center, and then reverting back into a pure liquid after the cavitation collapse. A similar test case was depicted in [31].

A uniform mesh of 5000 cells is used and the time step is set to $10^{-8} \mathrm{~s}$. The parameter $c_{\text {baro }}$ is set to $1.31 \mathrm{~m} / \mathrm{s}$ for the 4-equation barotropic model, as previously.

Volume fraction obtained with both models are plotted at different times in Fig. 5 . 
As the cavitation pocket grows, up to time $t=0.3 \mathrm{~ms}$, solutions are similar. After time $t=0.3 \mathrm{~ms}$, the shocks created at the ends meet the rarefaction waves generated at the center, and then interacts with the expanding cavitation interface. The cavitation collapse begins. The simulation obtained with the 4-equation barotropic model predicts the decrease of the void ratio. At time $t=0.7 \mathrm{~ms}$, the maximum void ratio value is close to 0.4. On the contrary, using the 4-equation SG model the decrease is badly reproduced. The maximum value at time $t=0.7 \mathrm{~ms}$ is 0.9 .

The 4-equation SG model is able to produce and to transport the void ratio but it does not destroy the void ratio in the flow where the pressure is greater than the vapour pressure. We propose to modify the formulation adding a destruction term:

$$
\dot{m}=\frac{\rho_{l} \rho_{v}}{\rho_{l}-\rho_{v}}\left(1-\frac{c^{2}}{c_{\text {wallis }}^{2}}\right) \operatorname{div} V-C_{\text {des }} \frac{\rho_{v}}{\rho_{l}} \alpha \frac{\operatorname{Max}\left(0, P-P_{\text {vap }}\right)}{0.5 \rho_{\text {ref }} U_{\text {ref }}^{2}}
$$

where $C_{d e s}$ is a tunable parameter. This new formulation does not modify the mixture speed of sound and the eigenvalues of the inviscid system.

The influence of the constant $C_{d e s}$ is investigated and different values are tested from 0.1 to 1000 . Using values smaller than 100 , the effect is weak and is not presented. The evolution of the volume fraction at different times is presented in Fig. 6 using

$C_{d e s}=100$ and $C_{d e s}=1000$. As expected, higher is the constant $C_{d e s}$, more intense is the condensation phenomenon. At time $t=0.7 \mathrm{~ms}$, the maximum void ratio value is 0.75 with $C_{d e s}=100$ and only 0.1 with $C_{\text {des }}=1000$.

\section{1D inviscid rarefaction test case with freon $\mathrm{R}-114$}

A double rarefaction tube problem is considered with freon R-114 similarly to those presented previously in water. The tube is filled with liquid freon at ambient tem- 
perature $T_{\text {ref }}=293 \mathrm{~K}$ and the pressure is set to 3 bar. A weak volume fraction of vapor $\alpha=0.01$ is initially added to the liquid. The initial discontinuity is set at $0.5 \mathrm{~m}$ and two velocities are tested: $u= \pm 10 \mathrm{~m} / \mathrm{s}$ and $u= \pm 100 \mathrm{~m} / \mathrm{s}$. The vapour pressure $P_{v a p}\left(T_{r e f}\right)$ is set to $181000 \mathrm{~Pa}$.

The mesh contains 5000 cells. The time step is set to $10^{-7} \mathrm{~s}$. The value of $c_{\text {baro }}$ is set to $1.66 \mathrm{~m} / \mathrm{s}$ for the 4-equation barotropic model.

The parameters of the stiffened gas EOS and saturation values for densities are given in Table 2. The quantities have been evaluated with a saturation table at the reference temperature.

\subsection{Velocity $|u|=10 \mathrm{~m} / \mathrm{s}$}

The value of $d P / d T$, evaluated with a thermodynamic table on the temperature interval $[283 K, 293 \mathrm{~K}]$, is set to $4720 \mathrm{~Pa} / \mathrm{K}$. Void ratio, velocity, temperature and pressure evolutions are shown in Fig. 7 at time $t=2.4 \mathrm{~ms}$. Both models provide a similar evolution for the void ratio and the velocity profiles. The pressure drop in the cavitation area is more pronounced with the 4-equation barotropic model. It is due to a higher temperature drop. It reaches $9 \mathrm{~K}$ with this model instead of $7 \mathrm{~K}$ with the 4-equation SG model. Using the B-factor theory and a maximum void ratio value equal to 0.8 , the ideal temperature depression is around $4.85 \mathrm{~K}$. Both models tend to overestimate this value.

\subsection{Velocity $|u|=100 \mathrm{~m} / \mathrm{s}$}

The value of $d P / d T$, evaluated with a thermodynamic table on the temperature interval $[50 K, 293 K]$, is set to $500 \mathrm{~Pa} / \mathrm{K}$. Void ratio, velocity, temperature and pressure evolutions are presented in Fig. 8 at time $t=1.5 \mathrm{~ms}$. Both models provide a similar 
evolution for the void ratio and the velocity profiles. As previously, the temperature depression is more intense using the 4-equation barotropic model. It reaches $260 \mathrm{~K}$ with this model and $240 \mathrm{~K}$ using the 4-equation SG model. This difference on the cooling effect leads to a more intense pressure drop in the cavitation pocket.

\section{2D viscous turbulent Venturi case}

\subsection{Experimental and numerical conditions}

The freon R-114 experimental facility of the CREMHyG is a closed loop operating with a reference pressure, obtained by pressurizing a tank with nitrogen gas. The cavitation tunnel was designed to simulate cavitating flows developing on the blades of space turbopump inducers. The loop is fitted with a test section having the shape of a two-dimensional Venturi, characterized by a convergence angle of $4.3^{\circ}$ and a divergence angle of $4^{\circ}$ (Fig. 9). The edge forming the throat of the Venturi is used to fix the separation point of the cavitation cavity. This geometry is equipped with three probing holes to take various measurements. Optical probes and micro-thermocouples are used to evaluate the local void ratio and the wall temperature, respectively. The uncertainty on the temperature measurement is about $\pm 0.2 \mathrm{~K}[43,44]$. Flow conditions and experimental parameters are given in Table 4. With these parameters, a cavity length around $80 \mathrm{~mm}$ was obtained, with a relatively stable aspect.

The freon R-114 is a thermosensitive fluid, which allows to study the thermodynamic effect in ambient conditions. It provides the same temperature depression $\Delta T^{*}=1.22 \mathrm{~K}$ in comparison with the liquid hydrogen at $T_{r e f}=22 \mathrm{~K}$. The thermodynamic properties at saturation for freon R-114 are given in Table 2. 
All cavitating simulations are steady computations, which are started from the non cavitating numerical solution. The H-type grid contains 251 nodes in the flow direction and 77 in the orthogonal direction (Fig. 10). Numerical parameters are given in Table 4.

Computations have been performed using both 4-equation models in order to obtain a cavity length close to $80 \mathrm{~mm}$. The value of the inlet cavitation parameter $\sigma_{\text {inlet }}$ was around 0.57 for all computations, close to the experimental value. In the following, the numerical solutions are compared with the previous results obtained with a 3equation model [24] and the experimental data.

\subsection{Visualization of the pocket}

A visualization of the cavitation pocket is proposed in Figure 11 where the contours of the density gradient modulus are plotted (Schlieren-like visualization). The grey scale is the same for all computations. The cavity length is around $80 \mathrm{~mm}$ for all simulations. Using the 3-equation model, the interface of the cavity and the closure part of the cavity are more diffuse in comparison with the 4-equation solutions. Discrepancies appear also on the thickness of the cavity. The 4-equation barotropic model predicts the thinner cavity.

\subsection{Void ratio profiles}

Figure 12 illustrates the numerical void ratio obtained with the three models in comparison with the measurements. At station 1, all models over-predict the cavity thickness and the maximum value of void ratio. According to analyses presented in [43], the experimental void ratio is certainly under-estimated at this station. At stations 2 and 3, the cavity thickness is clearly better predicted by the 4-equation 
barotropic model. Using the 3-equation model, the thickness is over-estimated with a factor 2. An intermediate result is provided by the 4-equation SG model. As regard to the maximum value of the void ratio, the 4-equation barotropic model predicts a higher value in comparison with the two other models. The decrease of the void ratio close to the wall is not captured by models. This behaviour was also observed for cold water simulations [32].

\subsection{Temperature evolution}

The temperature deficit profile at station 1 is plotted in Figure 13 for the three models. The wall temperature is in good agreement with the experimental data using the 3-equation model and the 4-equation barotropic model. Using the 4-equation SG model, the wall temperature depression is about $4.2 \mathrm{~K}$ instead of $2.1 \mathrm{~K}$ for the experimental data. Moreover, the shape of the temperature profile marks discrepancies. The temperature deficit is almost constant in a large part of the cavity from the wall up to $y=0.002 \mathrm{~m}$.

Figure 14 shows the temperature deficit $T-T_{\text {ref }}$ inside the divergent of the Venturi for the three computations. The grey scale is the same for all computations. The cooling effect due to the vaporization process is clearly observed for all simulations (negative values) and it is stronger using the 4-equation SG model. A large discrepancy appears between the 3- and 4-equation models downstream the cavity in the recompression area. Using the 3-equation model, the temperature deficit is close to zero downstream the cavitation pocket, that is the temperature goes back to the freestream temperature. On the contrary, we observe a warming effect downstream the cavity using both 4-equations models. Locally, the temperature exceeds the freestream temperature and values reach more than $6 \mathrm{~K}$ with the 4-equation $\mathrm{SG}$ 
model.

Such a phenomenon was depicted in [45] on a hot water Venturi flow. Using high speed infra-red thermography, authors measured a temperature depression of approximately $0.4 \mathrm{~K}$ in the vicinity of the throat and a temperature rise up to $1.4 \mathrm{~K}$ was recorded in the region of pressure recuperation. The collapse of bubbles causes the rapid recuperation of the temperature.

The same temperature difference is plotted in Figure 15 in a larger domain for the 4-equation barotropic model. The elevation of temperature is extended inside the divergent up to a distance of $0.4 \mathrm{~m}$ that is more than four times the cavity length.

The temperature gradient is illustrated in Figure 16 for the three computations. The grey scale is the same for all computations. We clearly observe differences on the behaviour of models in the closure part of the cavitation pocket. Using the 3-equation model, there is no temperature gradient at the end of the pocket. We just see a small gradient at the interface in the vicinity of the throat. With both 4-equation models, strong gradients are put in evidence in the closure part of the pocket and downstream in the recompression area.

\subsection{Influence of the destruction term}

The destruction term is now activated in the both 4-equation models following relation (32). This term is driven by a parameter $C_{d e s}$. Only one value $C_{\text {des }}=100$ was tested to observe the condensation process.

Figure 17 illustrates the void ratio and temperature deficit profiles computed with the 4-equation barotropic model. Profiles are given at different locations in the di- 
vergent: $x=0.065 \mathrm{~m}, x=0.075 \mathrm{~m}$ inside the pocket and $x=0.095 \mathrm{~m}, x=0.135 \mathrm{~m}$ downstream the pocket. At stations 1 to 3, void ratio and temperature profiles are not modified because the destruction term is not activated in the vaporization area. Inside the cavity at both abscissa $x=0.065 \mathrm{~m}$ and $x=0.075 \mathrm{~m}$, the effect of the destruction term is clearly noticeable on the void ratio maximum value, which is reduced by a factor 2. Similarly, the warming effect is also reduced (about $1 \mathrm{~K}$ ). At the abscissa $x=0.095 \mathrm{~m}$, the void ratio profiles provided by both simulations are quasi superposed. Yet, the temperature deficit profiles mark large discrepancies. The warming effect is well exhibited when $C_{\text {des }}=0$ whereas a cooling effect (about $1 \mathrm{~K}$ ) is predicted when the destruction term is activated. One possible explanation is linked to the transient of the computation. A large pocket for which the length reaches $0.2 \mathrm{~m}$ is simulated without the destruction term. In the closure part of the pocket an intense warming effect is predicted. When the cavity narrows, the warming effect remains a long time, which is observed at the abscissa $x=0.095 \mathrm{~m}$ and $x=0.135 \mathrm{~m}$. For this last location, with $C_{d e s}=100$ the void ratio is null and the temperature is on its freestream value. On the contrary, without the activation of the destruction term the warming effect is intense and reaches almost $8 \mathrm{~K}$.

At the same locations, the void ratio and temperature profiles computed with the 4-equation SG model are plotted in Figure 18. At both abscissa $x=0.065 \mathrm{~m}$ and $x=0.075 \mathrm{~m}$, the void ratio profiles provided by simulations are quasi superposed. The destruction of void ratio is not observed. As a consequence, the temperature deficit profiles are quite similar. At the abscissa $x=0.095 \mathrm{~m}$ and $x=0.135 \mathrm{~m}$, the same behaviour observed previously with the 4-equation batrotropic model is put in evidence. With similar void ratio profiles at location $x=0.095 \mathrm{~m}$, temperature evolutions are opposite: a warming effect (about $5 \mathrm{~K}$ ) without the destruction term 
and a cooling effect (around $1 \mathrm{~K}$ ) when the destruction term is used. At the last abscissa, a strong temperature elevation about $8 \mathrm{~K}$ is predicted when $C_{\text {des }}=0$. Using the destruction term, pure liquid at the freestream temperature is computed.

The temperature difference $T-T_{\text {ref }}$ is illustrated in Figure 19 for both simulations using the destruction term. We can observe the warming effect located on the closure part of the cavitation pocket. This phenomenon is not extended as previously simulated and the back to the freestream temperature is done on a short distance (less than one cavity length). Yet, as the experimental temperature field is unknown, it is impossible to calibrate the value of the parameter $C_{d e s}$.

\section{Conclusion}

In this paper, a 4-equation model was developed to study cavitation in thermosensitive fluid. This model is composed by three conservations law for mixture quantities (mass, momentum, total energy) and an additional transport equation for the vapour volume fraction, where mass transfer rate due to cavitation is modelled. The generic model is based on the assumption of proportionality of the mass transfer with the divergence of velocity. Two formulations were proposed using two equations of state: a modified barotropic relation and a mixture of stiffened gas. The vapour pressure is assumed to vary linearly with the mixture temperature through a parameter $d P_{v a p} / d T$. Models have been implemented in a compressible Euler and RANS solvers and have been applied for the simulation of various cavitating problems (inviscid and turbulent cases).

First validations on inviscid one-dimensional cases shown the ability of models to simulate the cavitation development in which the running fluid is hot water and

freon R-114. Comparisons with two-fluid solutions illustrated the good behaviour of 
models.

Secondly, RANS simulations were performed to study a quasi stable cavitation pocket developing along a Venturi geometry in which the working fluid is freon R-114. Numerical results obtained from the new models have been validated against experimental data and 3-equation computations previously performed in our team. This test-case lead to different concluding remark:

- About the cavity thickness, the new models and especially the 4-equation barotropic model clearly improved the prediction in comparison with the 3-equation simulation.

- The wall temperature deficit was well simulated by the 3- and 4-equation barotropic models. Yet, the solution obtained with the 4-equation SG model badly reproduced the wall value (over-estimation of a factor 2).

- A new phenomenon was simulated with the 4-equations models: a warming effect was exhibited downstream the cavity in the recompression area. The intensity of this warming effect is higher than the cooling effect observed near the Venturi throat. This rise of temperature is due to the collapse of bubbles in the closure part of the pocket and recent infra-red measurements highlighted this phenomenon inside another Venturi geometry in which the running fluid was hot water.

- The distance where this temperature elevation was simulated depends on the condensation modelling. Without the activation of a destruction term, the warming effect was extended more than 4 times the cavity length. Using a constant $C_{\text {des }}=100$ in the destruction term, the distance became smaller than one cavity length. Unfortunately, we need to know the temperature field to calibrate the destruction parameter. Finally, the new LEGI models are very attractive to study thermodynamic effects and cryogenic cavitation. Additional works are in progress to pursue comparative analyses between cavitation models and to improve the model calibration. 


\section{Appendix}

Appendix A: the speed of sound in a mixture of stiffened gas

Starting from the usual thermodynamic relation

$$
d e=T d s+\frac{P}{\rho^{2}} d \rho \quad \text { or } \quad d(\rho e)=\rho T d s+h d \rho
$$

And with the differential of $\rho e$ :

$$
d(\rho e)=\left(\frac{\partial \rho e}{\partial \rho}\right)_{P} d \rho+\left(\frac{\partial \rho e}{\partial P}\right)_{\rho} d P
$$

We can obtained the differential of the pressure $P$ :

$$
\left(\frac{\partial \rho e}{\partial P}\right)_{\rho} d P=\rho T d s+\left[h-\left(\frac{\partial \rho e}{\partial \rho}\right)_{P}\right] d \rho
$$

We deduce an expression of the speed of sound:

$$
c^{2}=\left(\frac{\partial P}{\partial \rho}\right)_{s}=\frac{h-\left(\frac{\partial \rho e}{\partial \rho}\right)_{P}}{\left(\frac{\partial \rho e}{\partial P}\right)_{\rho}}
$$

The derivatives of the void ratio have to be evaluated :

$$
\begin{aligned}
& \left(\frac{\partial \alpha}{\partial \rho}\right)_{P}=\frac{-1}{\rho_{L}-\rho_{V}} \\
& \left(\frac{\partial \alpha}{\partial P}\right)_{\rho}=\frac{-1}{\rho_{V}-\rho_{L}} \frac{d T}{d P}\left[\alpha \frac{d \rho_{V}}{d T}+(1-\alpha) \frac{d \rho_{L}}{d T}\right]
\end{aligned}
$$

Using the stiffened gas EOS, we have the following equalities:

$$
\begin{aligned}
\left(\frac{\partial \rho e}{\partial P}\right)_{\rho} & =\alpha\left(\frac{\partial \rho_{V} e_{V}}{\partial P}\right)_{\rho}+(1-\alpha)\left(\frac{\partial \rho_{L} e_{L}}{\partial P}\right)_{\rho}+\left(\rho_{V} h_{V}-\rho_{L} h_{L}\right)\left(\frac{\partial \alpha}{\partial P}\right)_{\rho} \\
& =\frac{1}{\gamma-1}+\frac{\rho_{V} h_{V}-\rho_{L} h_{L}}{\rho_{L}-\rho_{V}} \frac{d T}{d P}\left[\alpha \frac{d \rho_{V}}{d T}+(1-\alpha) \frac{d \rho_{L}}{d T}\right] \\
\left(\frac{\partial \rho e}{\partial \rho}\right)_{P} & =\frac{\partial}{\partial \rho}\left[\alpha\left(\frac{P}{\gamma_{V}-1}+\rho_{V} q_{V}+\frac{\gamma_{V}}{\gamma_{V}-1} P_{\infty}^{V}\right)+(1-\alpha)\left(\frac{P}{\gamma_{L}-1}+\rho_{L} q_{L}+\frac{\gamma_{L}}{\gamma_{L}-1} P_{\infty}^{L}\right)\right] \\
& =\frac{\rho_{L} h_{L}-\rho_{V} h_{V}}{\rho_{L}-\rho_{V}}
\end{aligned}
$$


Finally, the speed of sound is:

$$
\rho c^{2}=\frac{1}{\left(\frac{\partial \rho e}{\partial P}\right)_{\rho}}\left[\frac{\rho_{V} \rho_{L}}{\left(\rho_{L}-\rho_{V}\right)}\left(h_{V}-h_{L}\right)\right]
$$




\section{References}

[1] Saurel, R., Metayer, O.L.. A multiphase model for compressible flows with interfaces, shocks, detonation waves and cavitation. Journal of Fluid Mechanics 2001;431:239-271.

[2] Ishimoto, J., Kamijo, K.. Numerical study of cavitating flow characteristics of liquid helium in a pipe. Int Journal of Heat and Mass Transfer 2004;(47):149163.

[3] Metayer, O.L., Massoni, J., Saurel, R.. Modelling evaporation fronts with reactive Riemann solvers. Journal of Computational Physics 2005;205(2):567610.

[4] Yeom, G., Chang, K.. Numerical simulation of two-fluid two-phase flows by HLL scheme using an approximate jacobian matrix. Numerical Heat Transfer, Part B 2006;49:155-177.

[5] Zein, A., Hantke, M., Warnecke, G.. Modeling phase transition for compressible two-phase flows applied to metastable liquids. Journal of Computational Physics 2010;229(8):2964-2998.

[6] Mimouni, S., Archer, A., Laviville, J., Boucker, M., Mchitoua, N.. Modelling and computation of unsteady cavitation flows. La Houille Blanche 2006;6:121128.

[7] Mimouni, S., Boucker, M., Lavieville, J., Guelfi, A., Bestion, D.. Modelling and computation of cavitation and boiling bubbly flows with Neptune CFD code. Nuclear Engineering and Design 2008;238:680-692. 
[8] Kapila, A., Menikoff, R., Bdzil, J., Son, S., Stewart, D.. Two-phase modeling of deflagration-to-detonation transition in granular materials: reduced equations. Physics of fluids 2001;13(10):3002-3024.

[9] Petitpas, F., Franquet, E., Saurel, R., Metayer, O.L.. A relaxation-projection method for compressible flows. Part II: artificial heat exchanges for multiphase shocks. Journal of Computational Physics 2007;225(2):2214-2248.

[10] Saurel, R., Petitpas, F., Abgrall, R.. Modelling phase transition in metastable liquids: application to cavitating and flashing flows. Journal of Fluid Mechanics 2008;607:313-350.

[11] Hosangadi, A., Ahuja, V.. Numerical study of cavitation in cryogenic fluids. Journal of Fluids Engineering 2005;127(2):267-281.

[12] Utturkar, Y., Wu, J., Wang, G., Shyy, W.. Recent progress in modelling of cryogenic cavitation for liquid rocket propulsion. Progress in Aerospace Sciences 2005;41:558-608.

[13] Tseng, C., Shyy, W.. Modeling for isothermal and cryogenic cavitation. Int Journal of Heat and Mass Transfer 2010;53:513-525.

[14] Zhang, X., Qiu, L., Gao, Y., Zhang, X.. Computational fluid dynamic study on cavitation in liquid nitrogen. Cryogenics 2008;48:432-438.

[15] Goncalves, E., Patella, R.F.. Constraints on equation of state for cavitating flows with thermodynamic effects. Applied Math and Computation 2011;217:5095-5102. 
[16] Downar-Zapolski, P., Bilicki, Z., Bolle, L., Franco, J.. The non-equilibrium relaxation model for one-dimensional flashing liquid flow. Int Journal of Multiphase Flow 1996;22(3):473-483.

[17] Bilicki, Z., Kwidzinski, R., Mohammadein, S.. Evaluation of the relaxation time of heat and mass exchange in the liquid-vapour bubble flow. Int Journal of Heat and Mass Transfer 1996;39(4):753-759.

[18] Barret, M., Faucher, E., Herard, J.. Schemes to compute unsteady flashing flows. AIAA Journal 2002;40(5):905-913.

[19] Schmidt, D., Gopalakrishnan, S., Jasak, H.. Multi-dimensional simulation of thermal non-equilibrium channel flow. International Journal of Multiphase Flow 2010;36:284-292.

[20] Cooper, P.. Analysis of single and two-phase flow in turbopump inducers. Journal of Fluids Engineering 1967;89:577-588.

[21] Clerc, S.. Numerical simulation of the homogeneous equilibrium model for two-phase flows. Journal of Computational Physics 2000;161(1):354-375.

[22] Rapposelli, E., d'Agostino, L.. A barotropic cavitation model with thermodynamic effects. In: 5th International Symposium on Cavitation CAV2003, Osaka, Japan. 2003,.

[23] Rolland, J., Patella, R.F., Goncalves, E., Boitel, G., Barre, S.. Experiments and modelling of cavitating flows in venturi. Part I: stable cavitation. In: $6^{\text {th }}$ International Symposium on Cavitation CAV2006, Wageningen, The Netherlands. $2006,$. 
[24] Goncalves, E., Patella, R.F.. Numerical study of cavitating flows with thermodynamic effect. Computers \& Fluids 2010;39(1):99-113.

[25] Fujikawa, S., Okuda, M., Akamatsu, T.. Non-equilibrium vapour condensation on a shock-tube endwall behind a reflected shock wave. Journal of Fluid Mechanics 2080;(183):293-324.

[26] Franc, J.P., Pellone, C.. Analysis of thermal effects in a cavitating inducer using rayleigh equation. Journal of Fluid Engineering 2007;(129):974-983.

[27] Rodio, M., Giorgi, M.D., Ficarella, A.. Influence of convective heat transfer modeling on the estimation of thermal effects in cryogenic cavitating flows. Int Journal of Heat and Mass Transfer 2012;55:6538-6554.

[28] Florschuetz, L., Chao, B.. On the mechanics of vapor bubble collapse. Journal of Heat Transfer 1965;87:209-220.

[29] Prosperetti, A., Plesset, M.. Vapour-bubble growth in a superheated liquid. Journal of Fluid Mechanics 1978;85:349-368.

[30] Okhotsimskii, A.. The thermal regime of vapour bubble collapse at different jakob numbers. Int Journal of Heat and Mass Transfer 1988;31(8):1569-1576.

[31] Goncalves, E.. Numerical study of expansion tube problems: Toward the simulation of cavitation. Computers \& Fluids 2013;72:1-19.

[32] Goncalves, E., Charriere, B.. Modelling for isothermal cavitation with a fourequation modek. International Journal of Multiphase Flow 2014;59:54-72.

[33] Ishii, C., Hibiki, T.. Thermo-fluid dynamics of two-phase flow. Springer; 2006. 
[34] Metayer, O.L., Massoni, J., Saurel, R.. Elaborating equations of state of a liquid and its vapor for two-phase flow models. Int Journal of Thermal Sciences 2004;43:265-276.

[35] Wallis, G.. One-dimensional two-phase flow. New York: McGraw-Hill; 1967.

[36] Petitpas, F., Massoni, J., Saurel, R., Lapedie, E., Munier, L.. Diffuse interface model for high speed cavitating underwater systems. Int Journal of Multiphase Flow 2009;35:747-759.

[37] Spalart, P., Allmaras, S.. A one-equation turbulence model for aerodynamic flows. In: AIAA 92-0439, 30 ${ }^{\text {th }}$ Aerospace Sciences Meeting - Reno, Nevada. $1992,$.

[38] Goncalves, E., Decaix, J.. Wall model and mesh influence study for partial cavities. European Journal of Mechanics B/Fluids 2012;31(1):12-29.

[39] Jameson, A., Schmidt, W., Turkel, E.. Numerical solution of the Euler equations by finite volume methods using Runge-Kutta time stepping schemes. In: AIAA Paper 81-1259. 1981,.

[40] Roe, P.. Approximate Riemann solvers, parameters vectors, and difference schemes. Journal of Computational Physics 1981;43:357-372.

[41] Tatsumi, S., Martinelli, L., Jameson, A.. Flux-limited schemes for the compressible Navier-Stokes equations. AIAA Journal 1995;33(2):252-261.

[42] Franc, J.P., Michel, J.M.. Fundamentals of cavitation. Springer; 2004.

[43] Stutz, B.. Analyse de la structure diphasique et instationnaire de poches de cavitation. Ph.D. thesis; Institut National Polytechnique de Grenoble; 1996. 
[44] Fruman, D., Reboud, J., Stutz, B.. Estimation of thermal effects in cavitation of thermosensible liquids. Int Journal of Heat and Mass Transfer 1999;(42):31953204 .

[45] Petkovsek, M., Dular, M.. IR measurements of the thermodynamic effects in cavitating flow. International Journal of Heat and Fluid Flow 2013;44:756-763. 
Table 1: Characteristics of models

\begin{tabular}{|c|c|c|c|c|}
\hline models & 2-fluid & 4-eqt baro & 4-eqt SG & 3-eqt baro \\
\hline solved & 2 masses & 1 mass & 1 mass & 1 mass \\
\hline \multirow[t]{3}{*}{ equations } & 1 moment. & 1 moment. & 1 moment. & 1 moment. \\
\hline & 2 energies & 1 energy & 1 energy & 1 energy \\
\hline & $+\alpha$ & $+\alpha$ & $+\alpha$ & \\
\hline mixture EOS & $\mathrm{SG}$ & baro & $\mathrm{SG}$ & baro \\
\hline source term & $\mu\left(P_{l}-P_{v}\right)+\theta\left(T_{v}-T_{l}\right)$ & $K d i v V$ & $K d i v V$ & analytical \\
\hline$\alpha$ equation & $+\dot{m} / \rho_{I}$ & $+\dot{m} / \rho_{I}$ & $+\dot{m} / \rho_{I}$ & $\alpha=\frac{\left(\rho-\rho_{l}\right)}{\left(\rho_{v}-\rho_{l}\right)}$ \\
\hline$\dot{m}$ & $\nu\left(g_{v}-g_{l}\right)$ & $\left(1-\frac{c^{2}}{c_{w a l l i s}^{2}}\right) d i v V$ & $\left(1-\frac{c^{2}}{c_{w a l l i s}^{2}}\right) d i v V$ & - \\
\hline metastable & liquid & - & - & - \\
\hline states & vapour & vapour & vapour & - \\
\hline
\end{tabular}


Table 2: Parameters of the stiffened gas EOS for freon R114 at $T=293 \mathrm{~K}$.

\begin{tabular}{|c|c|c|c|c|c|}
\hline & $\gamma$ & $P_{\infty}(\mathrm{Pa})$ & $\mathrm{q}(\mathrm{J} / \mathrm{kg})$ & $C_{p}(\mathrm{~J} / \mathrm{K} . \mathrm{kg})$ & $\rho_{\text {sat }}\left(\mathrm{kg} / \mathrm{m}^{3}\right)$ \\
\hline liquid & 1.4 & $1.21 \quad 10^{8}$ & $-6.90110^{4}$ & 984 & 1469.1 \\
\hline vapor & 1.07 & 0 & $1.42410^{5}$ & 700 & 13.52 \\
\hline
\end{tabular}


Table 3: Parameters of the stiffened gas EOS for water at $T=355 \mathrm{~K}$.

\begin{tabular}{|c|c|c|c|c|c|}
\hline & $\gamma$ & $P_{\infty}(\mathrm{Pa})$ & $\mathrm{q}(\mathrm{J} / \mathrm{kg})$ & $C_{p}(\mathrm{~J} / \mathrm{K} . \mathrm{kg})$ & $\rho_{\text {sat }}\left(\mathrm{kg} / \mathrm{m}^{3}\right)$ \\
\hline liquid & 2.35 & $10^{9}$ & $-0.116710^{7}$ & 4267 & 1149.9 \\
\hline vapor & 1.43 & 0 & $0.203010^{7}$ & 1487 & 0.31 \\
\hline
\end{tabular}


Table 4: Flow configuration, experimental and numerical parameters, Venturi case

\begin{tabular}{lc} 
inlet velocity $V_{\text {inlet }}$ & $14.4 \mathrm{~m} / \mathrm{s}$ \\
inlet pressure $P_{\text {inlet }}$ & $265300 \mathrm{~Pa}$ \\
reference temperature $T_{r e f}$ & $\simeq 293 \mathrm{~K}$ \\
vapour pressure at $T_{r e f}$ & 181100 \\
cavitation parameter in the inlet section $\sigma_{\text {inlet }}=\frac{P_{\text {inlet }}-P_{\text {vap }}\left(T_{r e f}\right)}{0.5 \rho V_{\text {inlet }}^{2}}$ & $\simeq 0.55$ \\
reference length $L_{r e f}$ & $0.252 \mathrm{~m}$ \\
Reynolds number $R e_{L_{r e f}}=\frac{V_{\text {inlet }} \times L_{r e f}}{\nu\left(T_{r e f}\right)}$ & $18.410^{6}$ \\
\hline mesh & $251 \times 77$ \\
$y^{+}$values in first cells & 35 to 50 \\
constant $d P_{\text {vap }} / d T$, evaluated on the interval $[290,293] \mathrm{K}$ & $5900 \mathrm{~Pa} / \mathrm{K}$ \\
parameter $c_{\text {baro }}$ & $0.74 \mathrm{~m} / \mathrm{s}(\mathrm{see}[24])$ \\
CFL number & 0.5 \\
implicit Jacobi iterations & 15 \\
2nd and 4 th order dissipation parameter & $1 ; 0.04$ \\
\hline
\end{tabular}




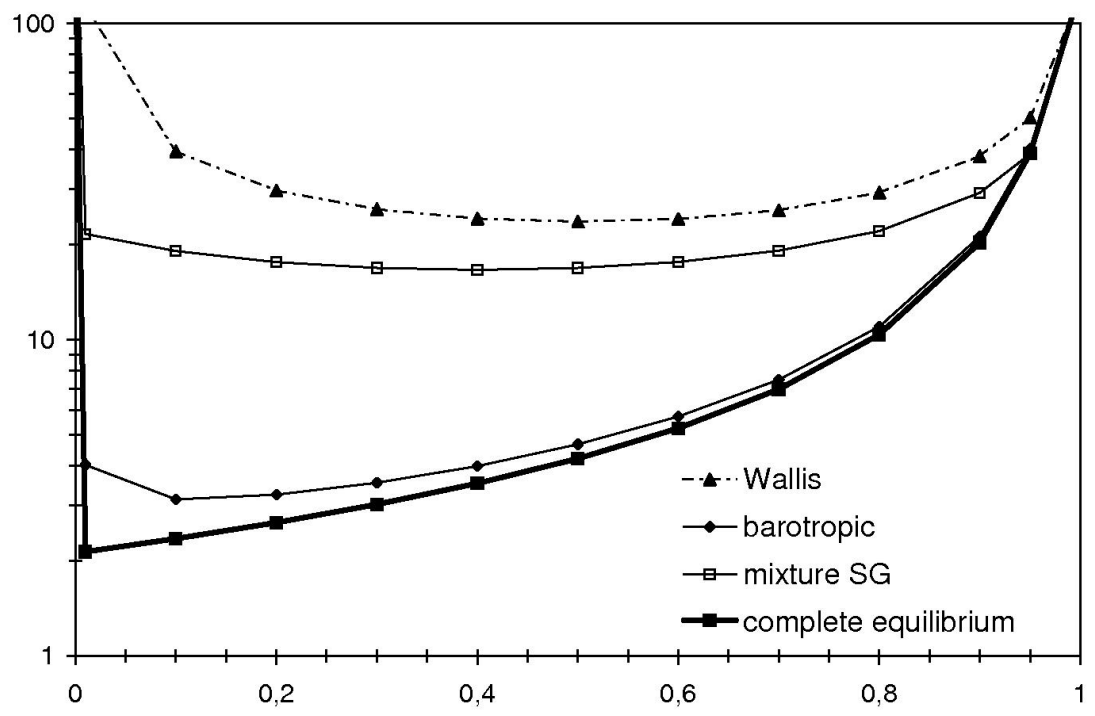

Figure 1: Mixture speed of sound comparison, freon R-114. 

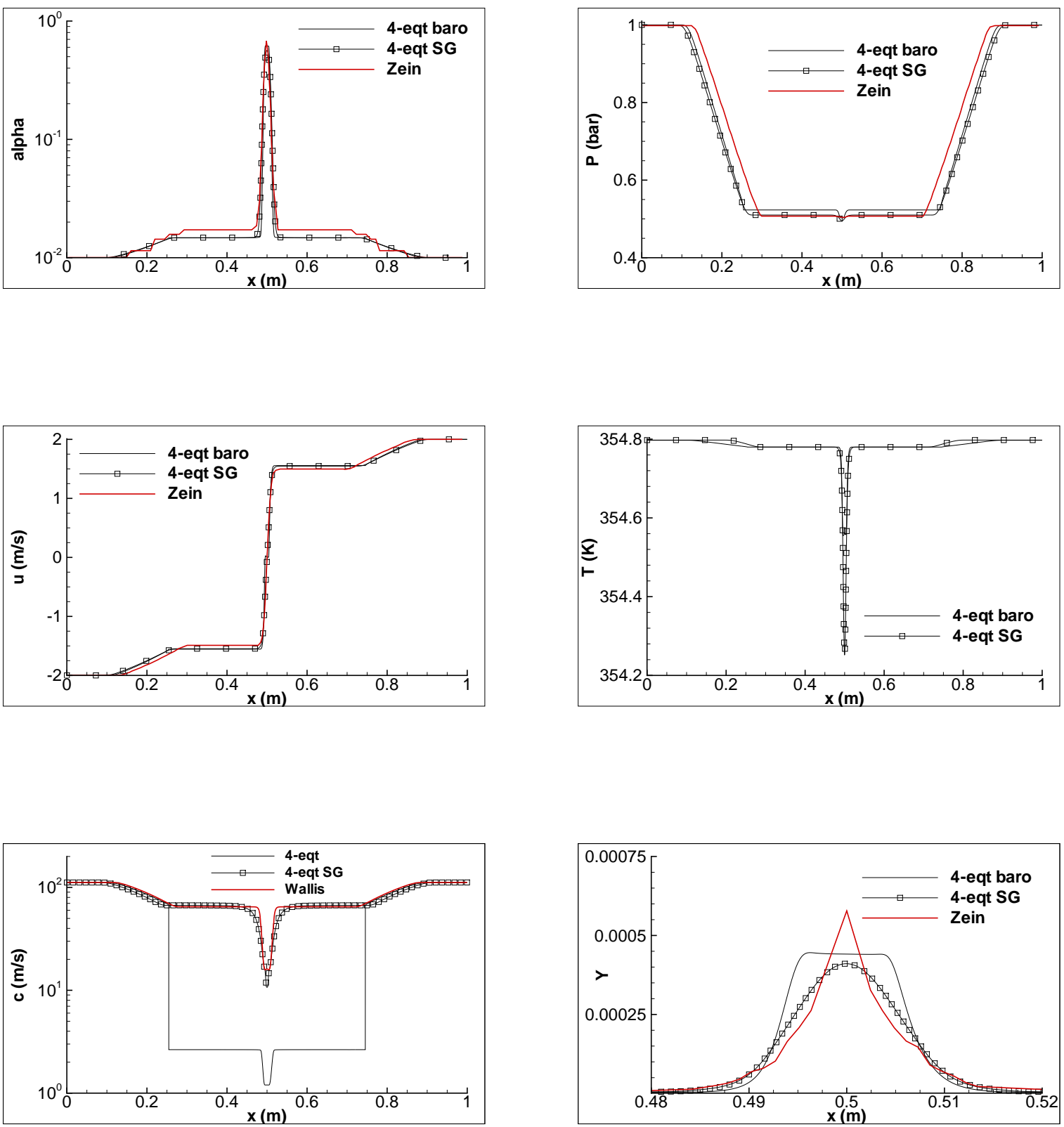

Figure 2: Water-gas double rarefaction with cavitation $|u|=2 \mathrm{~m} / \mathrm{s}$, models comparison, mesh 5000 cells, $t=3.2 \mathrm{~ms}$. Void ratio, pressure, velocity, temperature, speed of sound and mass fraction of gas. 

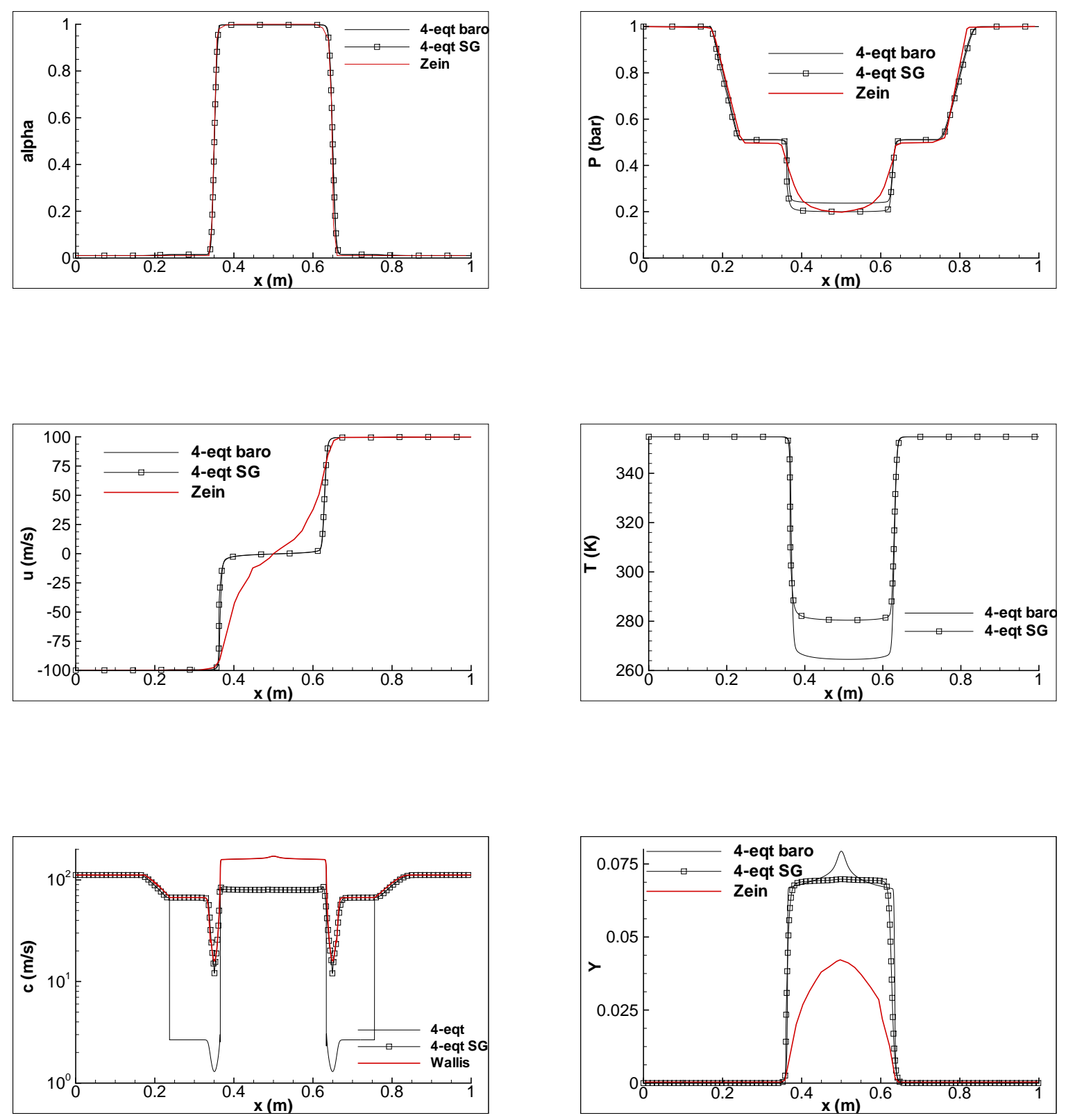

Figure 3: Water-gas double rarefaction with cavitation $|u|=100 \mathrm{~m} / \mathrm{s}$, models comparison, mesh 5000 cells, $t=1.5 \mathrm{~ms}$. Void ratio, pressure, velocity, temperature, speed of sound and mass fraction of gas. 

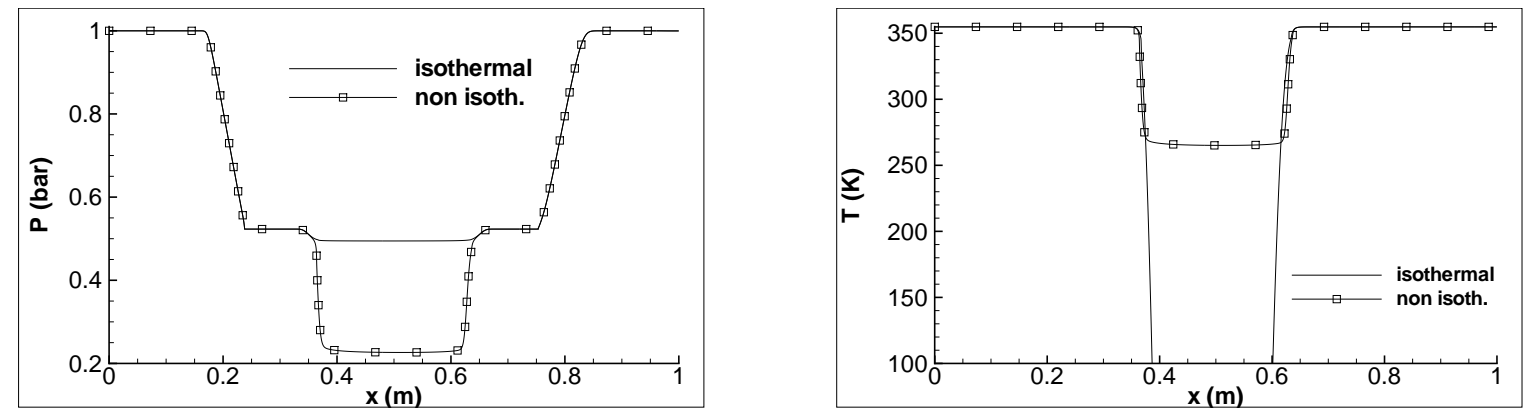

Figure 4: Water-gas double rarefaction with cavitation $|u|=100 \mathrm{~m} / \mathrm{s}$, isothermal versus non isothermal models, mesh 5000 cells, $t=1.5 \mathrm{~ms}$. Pressure and temperature. 

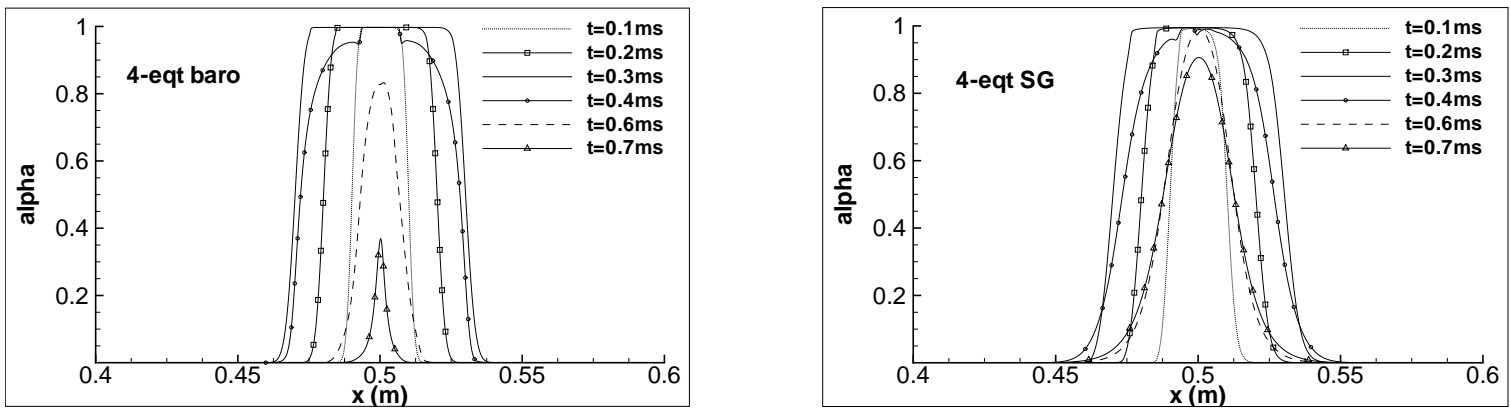

Figure 5: Shock-cavitation interaction $|u|=100 \mathrm{~m} / \mathrm{s}$, models comparison, mesh 5000 cells. Void ratio at different times. 

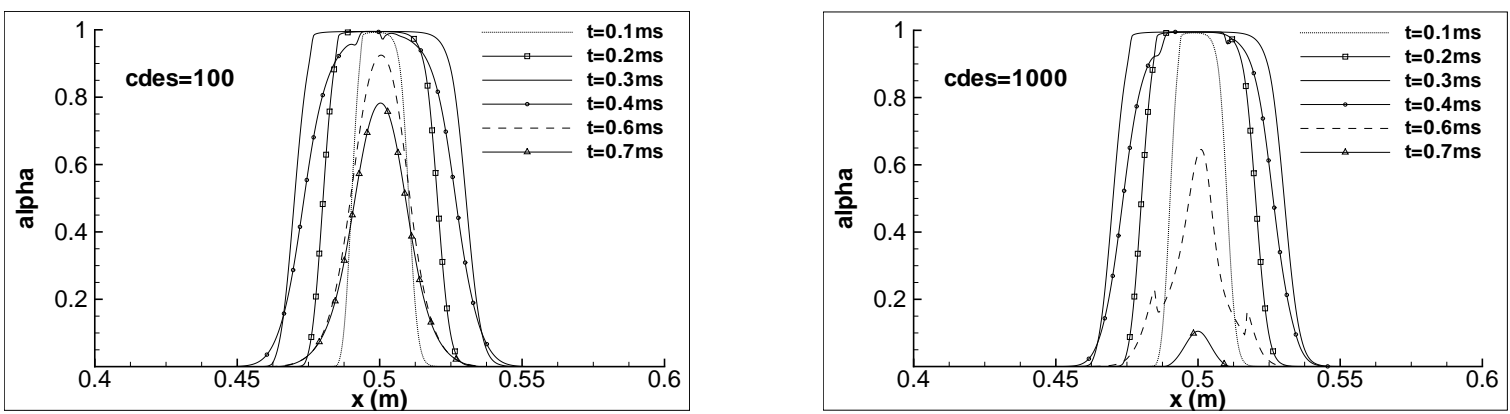

Figure 6: Shock-cavitation interaction $|u|=100 \mathrm{~m} / \mathrm{s}$, influence of $C_{d e s}$, 4-equation SG model, mesh 5000 cells. Void ratio at different times. 

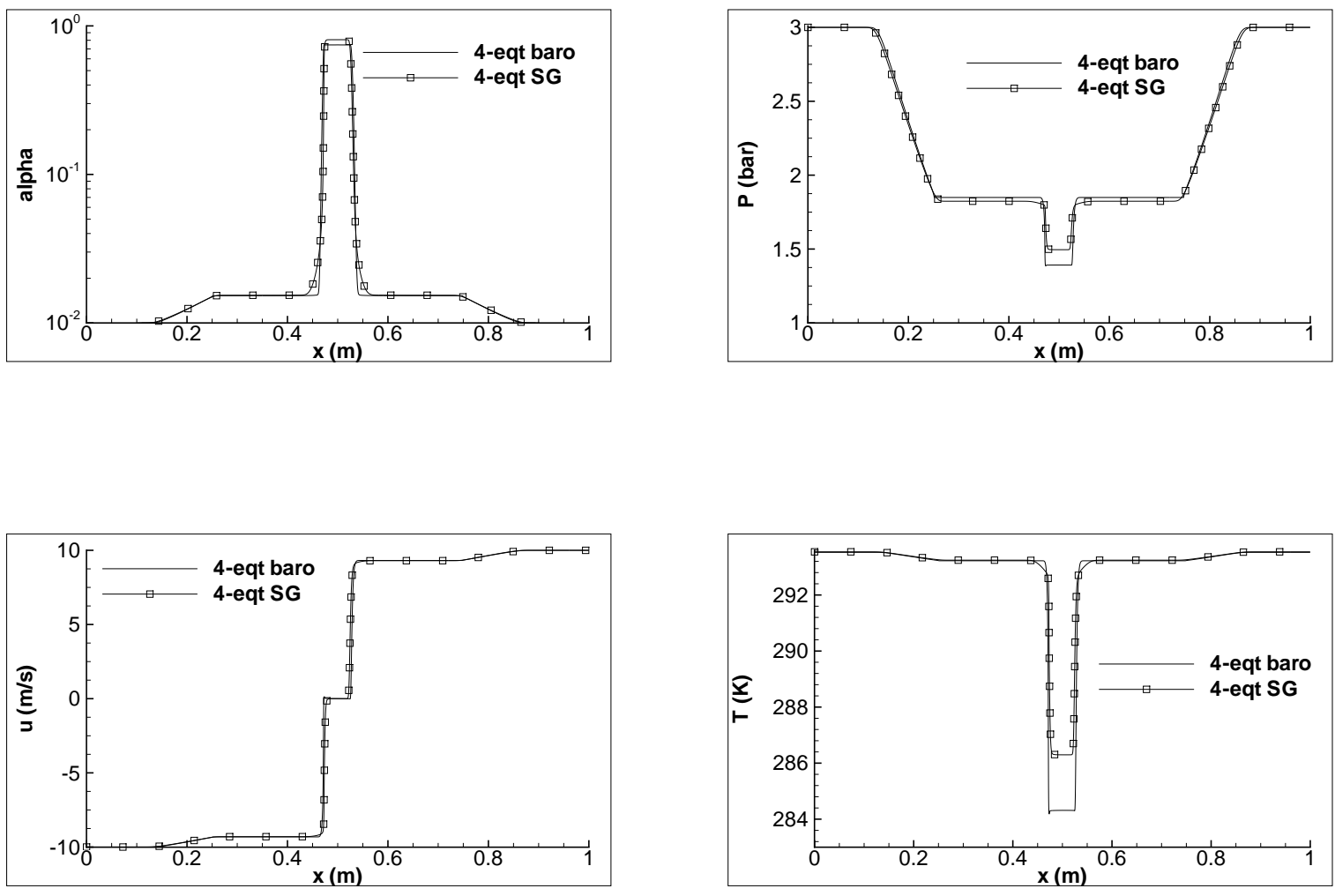

Figure 7: Freon R114 double rarefaction with cavitation $|u|=10 \mathrm{~m} / \mathrm{s}$, models comparison, mesh 5000 cells, $t=2.4 \mathrm{~ms}$. Void ratio, mixture pressure, temperature and velocity. 

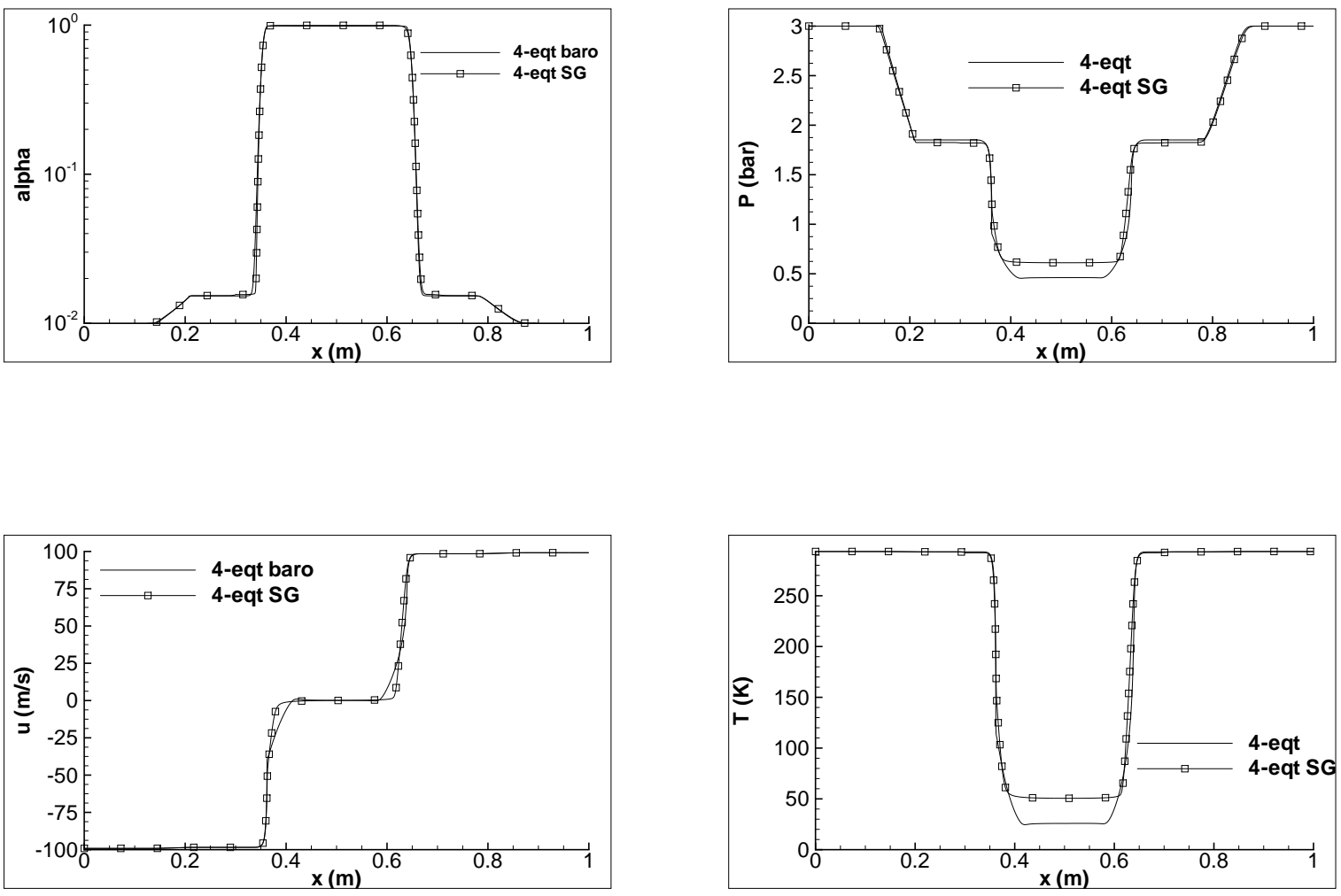

Figure 8: Freon R114 double rarefaction with cavitation $|u|=100 \mathrm{~m} / \mathrm{s}$, models comparison, mesh 5000 cells, $t=1.5 \mathrm{~ms}$. Void ratio, mixture pressure, temperature and velocity. 


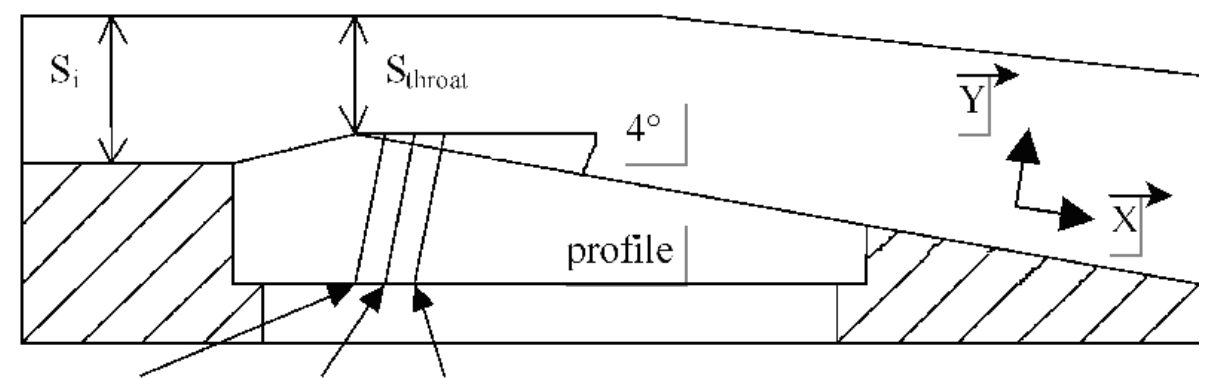

Station 1 Station 2 Station 3

Figure 9: Schematic view of the Venturi profile. 


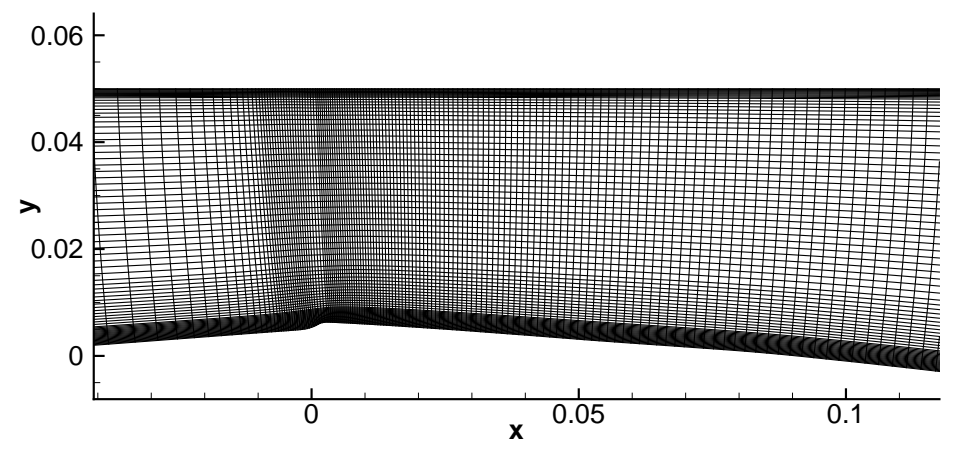

Figure 10: Enlargement of the mesh near the Venturi throat. 


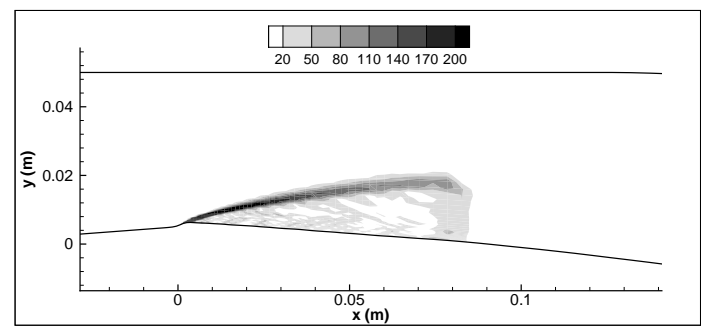

3-equation

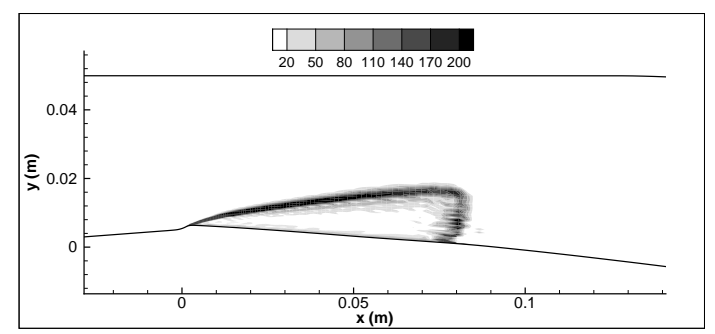

4-equation barotropic

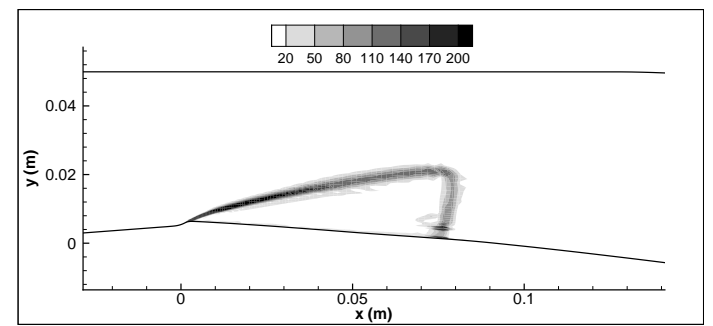

4-equation $\mathrm{SG}$

Figure 11: Modulus of the density gradient $\left(\mathrm{kg} / \mathrm{m}^{4}\right)$, models comparison, Venturi case. 

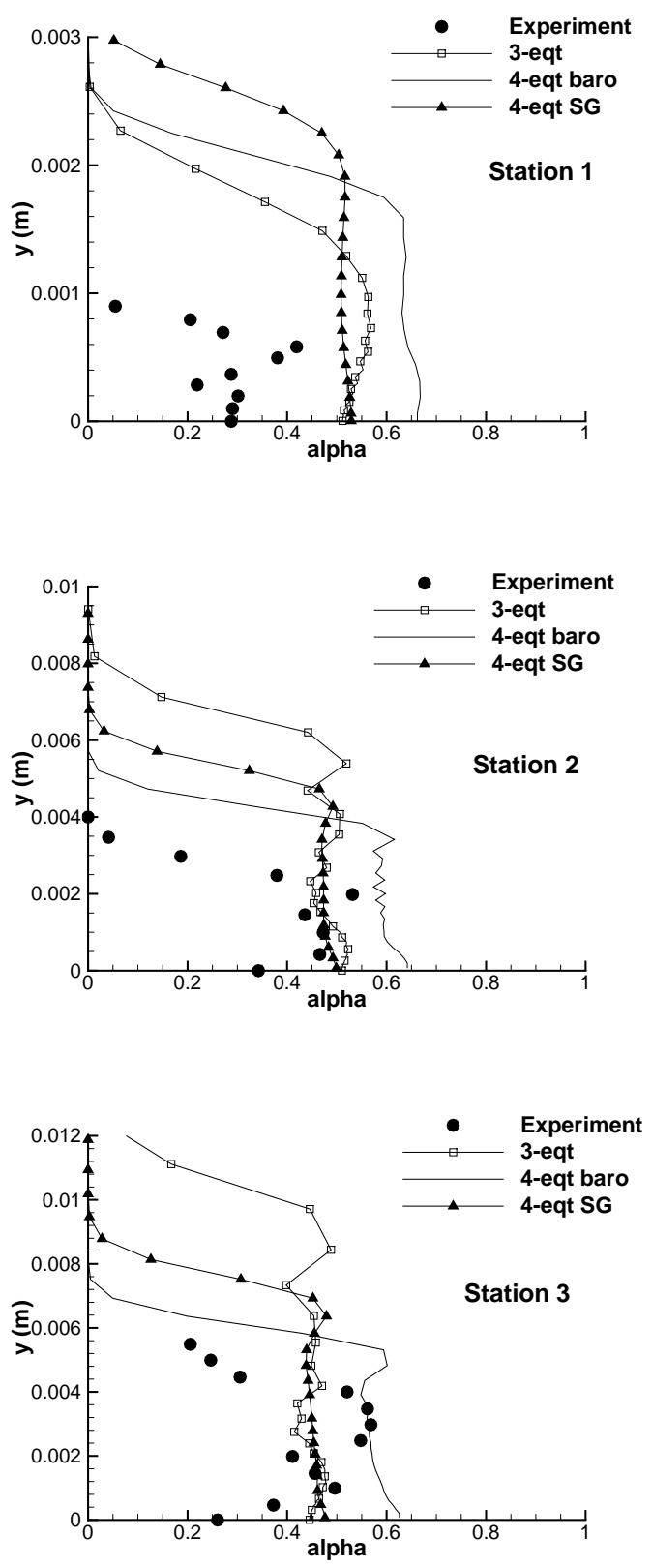

Figure 12: Void ratio profiles at stations 1 to 3, models comparison, Venturi case. 


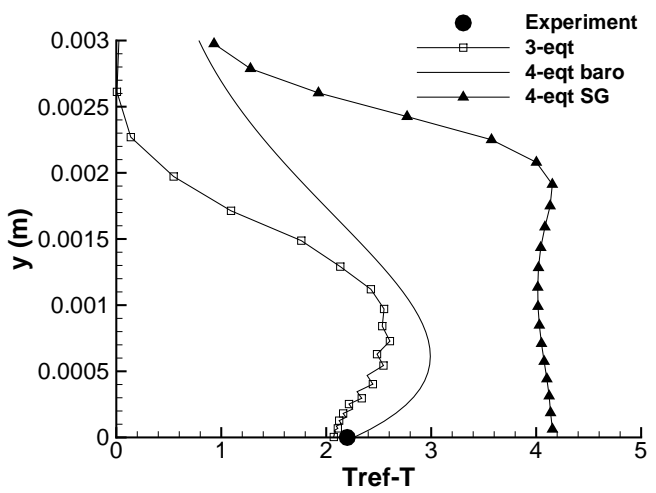

Figure 13: Wall temperature depression at station 1, models comparison, Venturi case. 


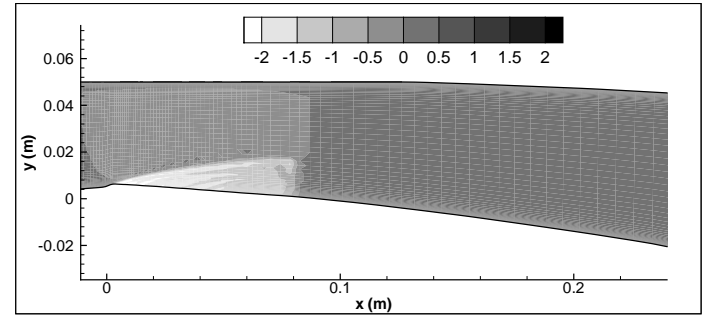

3-equation

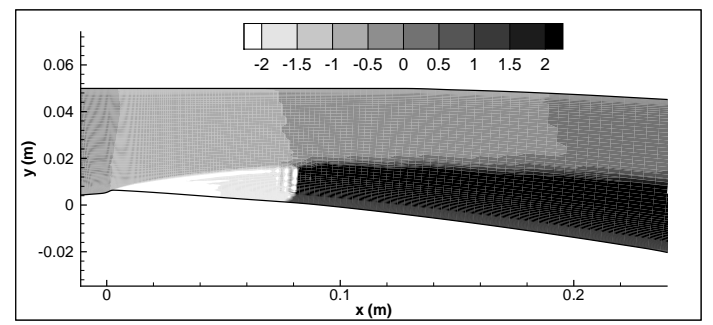

4-equation barotropic

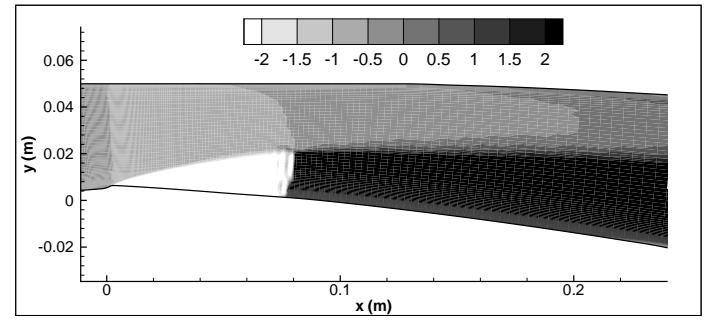

4-equation SG

Figure 14: Temperature difference $T-T_{r e f}(\mathrm{~K})$ inside the divergent, models comparison, Venturi case. 


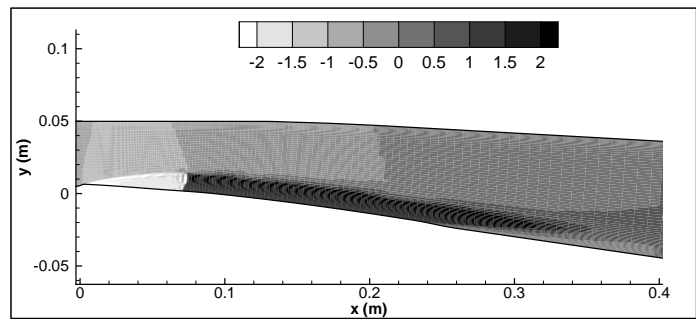

Figure 15: Temperature difference $T-T_{r e f}(\mathrm{~K})$ inside the divergent, 4-equation barotropic model, Venturi case. 


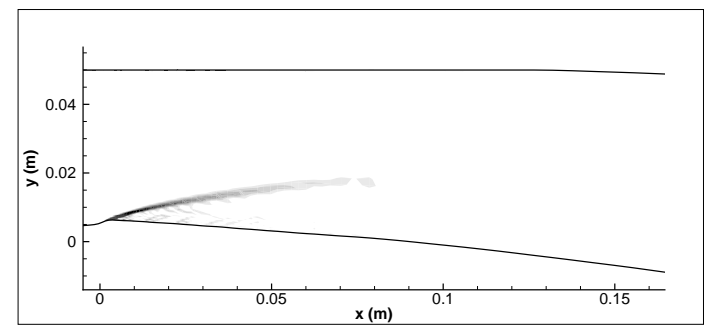

3-equation

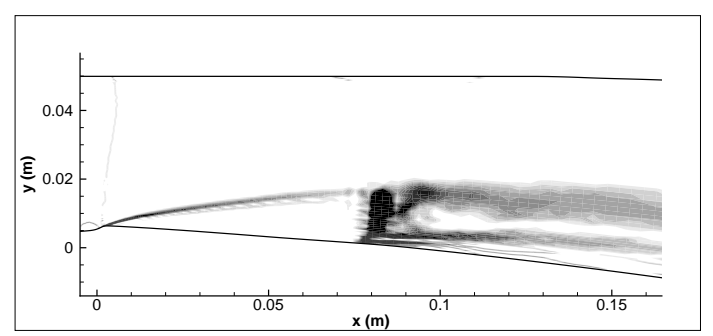

4-equation barotropic

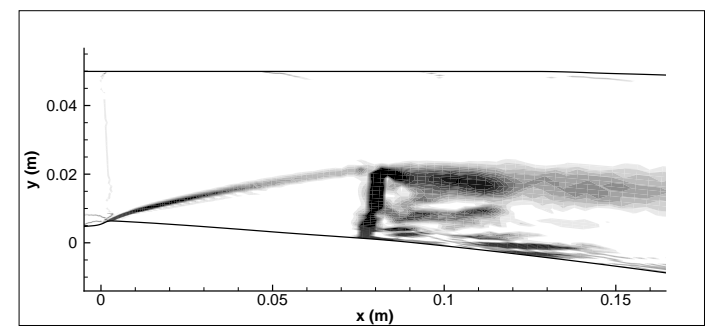

4-equation $\mathrm{SG}$

Figure 16: Temperature gradient $(\mathrm{K} / \mathrm{m})$ inside the divergent, models comparison, Venturi case. 

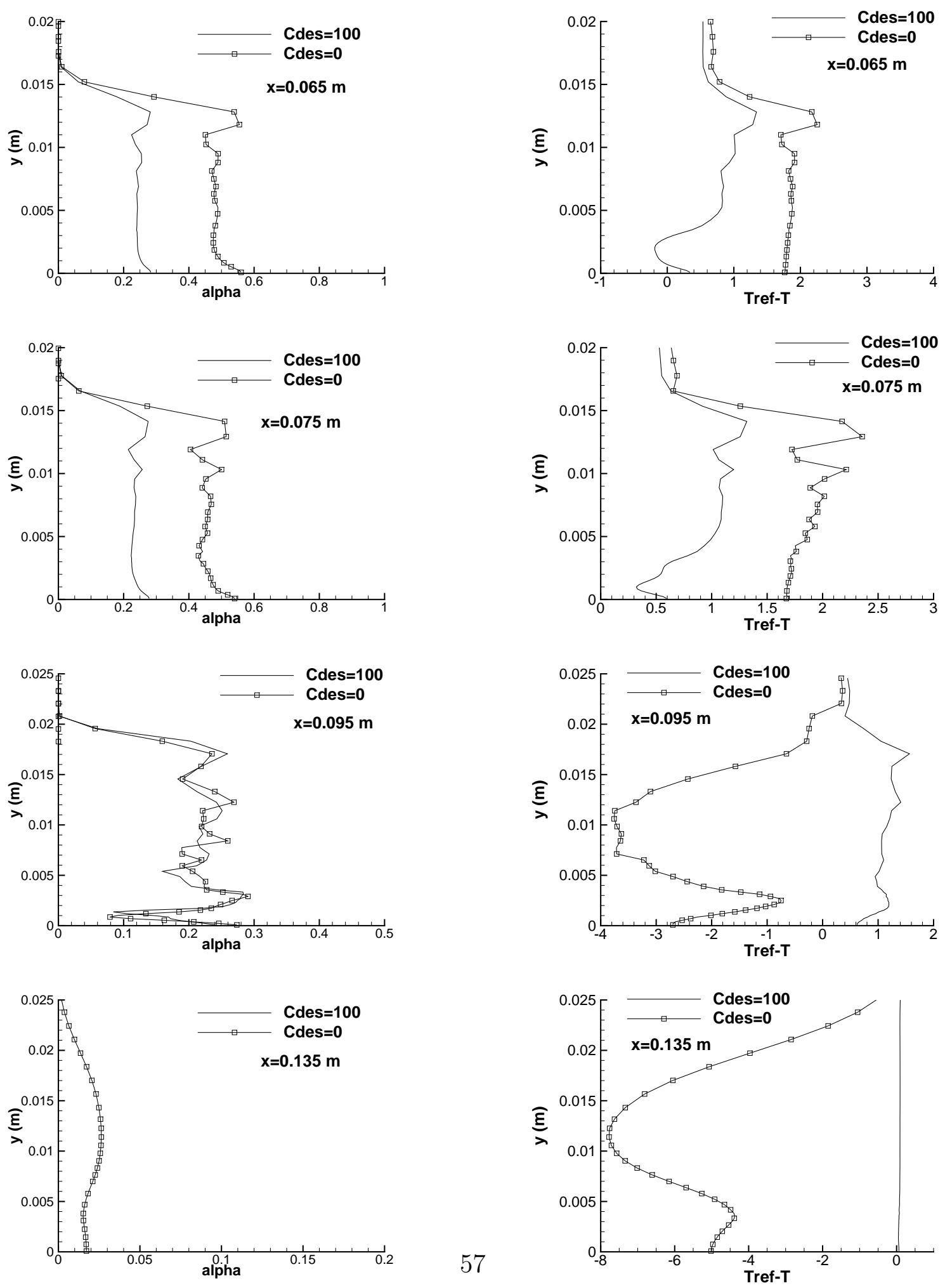

Figure 17: Void ratio (left) and temperature deficit (right) profiles at different locations, influence of the destruction term, 4-equation barotropic model, Venturi case. 

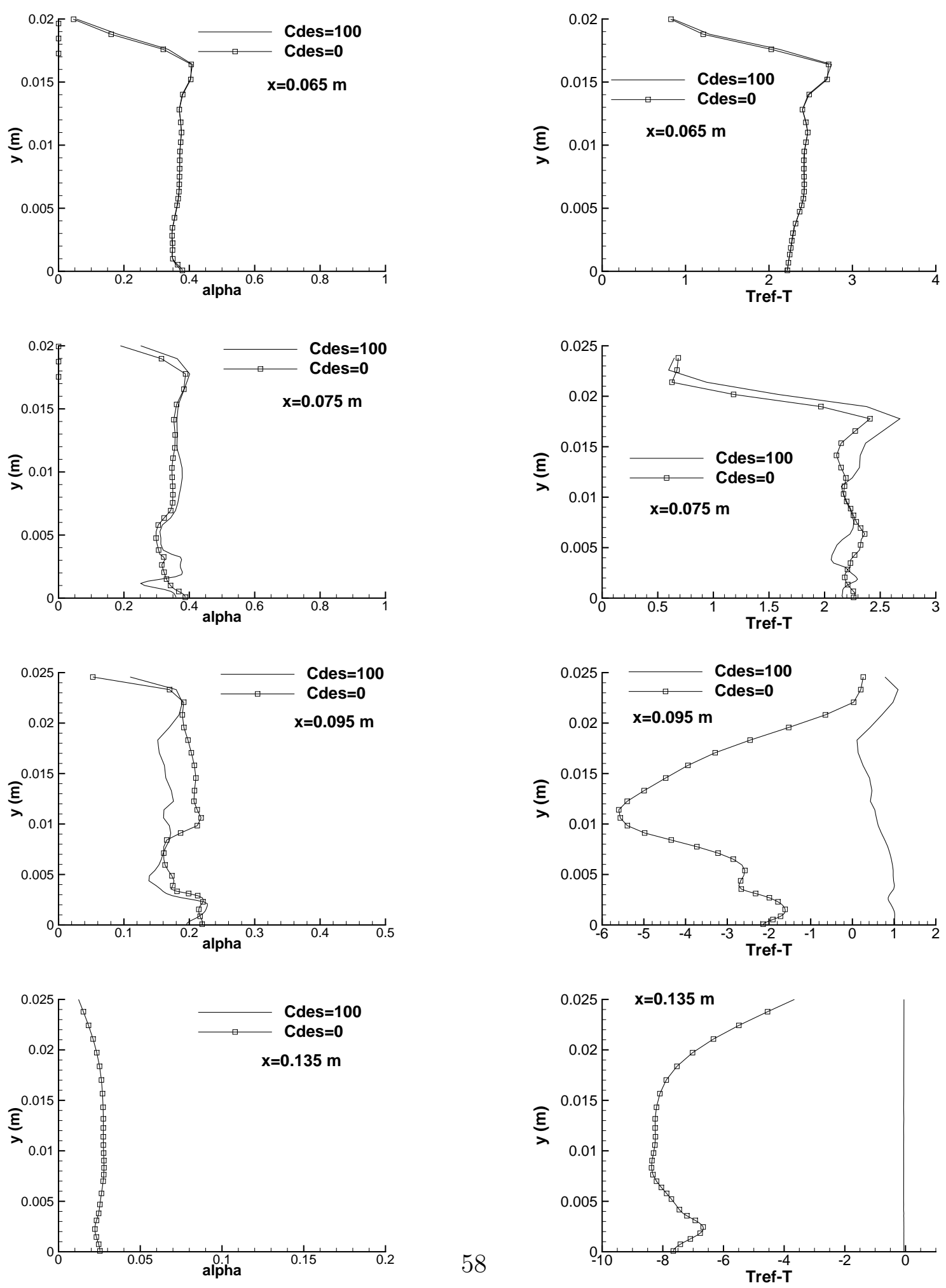

Figure 18: Void ratio (left) and temperature deficit (right) profiles at different locations, influence of the destruction term, 4-equation SG model, Venturi case. 


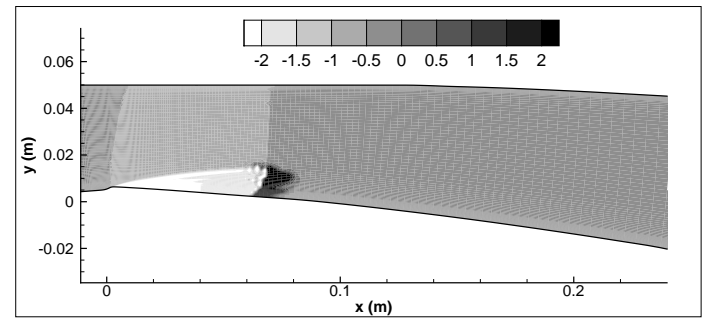

4-equation barotropic

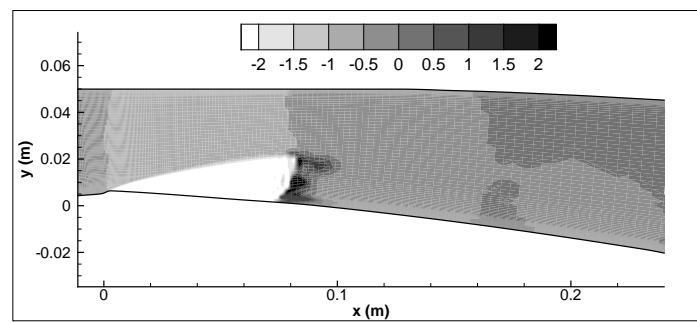

4-equation SG

Figure 19: Temperature difference $T-T_{\text {ref }}(\mathrm{K})$ inside the divergent, influence of the destruction term, Venturi case. 